\title{
Postcranial skeleton of Spalacopus cyanus (Rodentia: Octodontidae): description and functional aspects
}

\author{
M. Julieta Pérez ${ }^{1,2, *}$, and M. Mónica Díaz $z^{1,2,3}$ \\ 1 Programa de Investigaciones de Biodiversidad Argentina (PIDBA) y Programa de Conservación de los Murciélagos de Argentina \\ (PCMA), Facultad de Ciencias Naturales e Instituto Miguel Lillo, Universidad Nacional de Tucumán, Miguel Lillo 205, CP 4000. \\ San Miguel de Tucumán, Argentina. Email: mariju perez@hotmail.com (MJP), mmonicadiaz@yahoo.com.ar (MMD). \\ ${ }^{2}$ Consejo Nacional de Investigaciones Científicas y Técnicas (CONICET), Tucumán, Argentina. \\ ${ }^{3}$ Fundación Miguel Lillo, Tucumán, Argentina \\ *Corresponding autor
}

The genus Spalacopus includes only one species, S. cyanus, endemic to central Chile and one of the species best adapted to a subterranean lifetyle in the family Octodontidae. It is a member of the lineage consisting in Octodontomys as a sister clade containing Octodon and of a clade represented by Spalacopus and Aconaemys. Although the external and cranio-dental morphology have been well studied, little is known of the postcranial morphology of S. cyanus. Because of the peculiarities of its lifestyle and locomotion among the members of the family, it is interesting to characterize details of the morphology of the postcranial skeleton of this species and to infer their functional aspects of the morphology. Twenty-nine specimens with postcranial material stored in two collections were studied: Museo de La Plata (MLP), La Plata, Buenos Aires, Argentina and Colección de Mamíferos del Instituto de Ciencias Ambientales y Evolutivas (UACH), Universidad Austral de Chile, Valdivia, Chile. To describe in detail the morphology of the bone elements, the skeleton was divided in the following regions: axial skeleton, scapular girdle and forelimb, and pelvis girdle and hindlimb. The structures included in the axial skeleton were mainly described in cranial-caudal orientation and the limbs in proximal-distal orientation. All structures were observed with stereoscopic microscopes Leica Wild M3Z and Nikon SMZ 745T including pictures to illustrate the descriptions. Highly conservative characteristics among members of the family Octodontidae were observed in the postcranial morphology of S. cyanus, although some characters are exclusive to this species. The axial skeleton has seven cervical vertebrae, 12 or 13 thoracic, six or seven lumbar, four sacral, 16 to 18 caudal vertebrae, 12 or 13 ribs and a sternum with five sternebrae. The elements of the scapular and pelvic girdles as well as forelimbs and hindlimbs are described in detail, except the carpus and tarsus due to their poor state of conservation, or because they not available. The morphological pattern observed in the postcranial skeleton of $S$. cyanus conforms to a typical anatomical plan for terrestrial species, and some bones (mainly humerus and ulna) present characteristics associated with digging. The information obtained provided will allow a better interpretation of the postcranial attributes, functionally related with different lifestyle, in a future, as well as the evolution of the traits in a phylogenetic context.

El género Spalacopus incluye una sola especie, S. cyanus, endémica de la región central del Chile, siendo una de las especies mejor adaptada a la vida subterránea de la familia Octodontidae. Miembro del linaje que consiste en Octodontomys como hermano de Octodon y el clado representado por Spalacopus y Aconaemys. Si bien la morfología externa y cráneo-dentarias en S. cyanus han sido bien estudiadas, su morfología postcraneal es pobremente conocida. Por las peculiaridades de su estilo de vida y locomoción entre los miembros de la familia, es interesante caracterizar detalles de la morfología del esqueleto postcraneal de esta especie e inferir sus aspectos funcionales de la morfología. Se revisaron 29 ejemplares con material de postcráneo disponibles en dos colecciones sistemáticas: Museo de La Plata (MLP), La Plata, Buenos Aires, Argentina y Colección de Mamíferos del Instituto de Ciencias Ambientales y Evolutivas (UACH), Universidad Austral de Chile, Valdivia, Chile. Para la descripción detallada de la morfología de los elementos óseos se dividió al esqueleto en las siguientes regiones: esqueleto axial, cintura escapular y miembros anteriores, y cintura pélvica y miembros posteriores. Las estructuras incluidas en el esqueleto axial fueron descriptas principalmente en sentido cráneo-caudal y en los miembros en sentido próximo-distal. La observación de las estructuras se realizó con las lupas estereoscópicas Leica Wild M3Z y Nikon SMZ 745T, incluyendo fotografías para ilustrar las descripciones. En la morfología postcraneal de $S$. cyanus se observan características que se consideran altamente conservadas entre los miembros de la familia Octodontidae, pero algunos caracteres son exclusivos de la especie. El esqueleto axial está compuesto por siete vértebras cervicales, 12 ó 13 torácicas, seis o siete lumbares, cuatro sacras, entre 16 y 18 caudales, 12 ó 13 pares de costillas y un esternón compuesto por cinco esternebras. Los elementos óseos de las cinturas pectoral y pélvica como de los miembros anterior y posterior se describen en detalle, excepto los carpos y tarsos debido al mal estado de preservación o ausencia de los mismos. El patrón morfológico que presenta el postcráneo de S. cyanus se ajusta a un plan anatómico típico de especies terrestres y en algunos elementos óseos (húmero y ulna principalmente) se observan características típicamente asociadas con la habilidad excavadora. Los datos generados permitirán a futuro una mejor interpretación de los atributos postcraneales funcionalmente relacionados con los diferentes estilos de vida, evaluar sus estrategias locomotoras, así como también comprender la evolución de estos rasgos en un contexto filogenético.

Keywords: Chile, coruro, fuctional morphology, South America; subterranean rodent.

(C) 2020 Asociación Mexicana de Mastozoología, www.mastozoologiamexicana.org 


\section{Introducción}

Octodontidaees una familia de roedores de tamaño mediano (100 g in Octomys to $300 \mathrm{~g}$ in Octodon), restringidas al sur de Sudamérica, entre los $-15^{\circ}$ y $-43^{\circ} \mathrm{S}$ de latitud (Reig 1989; Gallardo et al. 2007; Ojeda et al. 2013; Verzi et al. 2015). Se distribuyen en Argentina, Bolivia y Chile, en una gran diversidad de hábitats (Gallardo et al. 2007).

Dentro de Octodontidae se pueden reconocer dos linajes principales, el grupo de las ratas-vizcachas, que consiste en una división basal de Octomys, del grupo que contiene a Tympanoctomys y el otro linaje que consiste en Octodontomys como hermano de Octodon (Spalacopus, Aconaemys; Upham y Patterson 2015; SuárezVillota et al. 2016). Las relaciones entre y dentro de algunos géneros permanecen sin resolver, como el caso de Spalacopus, Aconaemys y Tympanoctomys (Gallardo y Mondaca 2002; Honeycutt et al. 2003; Upham y Patterson 2012, 2015; Suárez-Villota et al. 2016).

La capacidad para cavar es prevalente en Octodontidae al igual que en la familia hermana, Ctenomyidae (Lessa et al. 2008). Además, entre los octodóntidos, el linaje que conduce a Spalacopus evolucionó a una vida completamente subterránea con la adquisición de numerosos cambios morfológicos alrededor de $2.2 \mathrm{Ma}$ (Lessa et al. 2008); mientras que en ctenómidos tienen cambios acumulados asociados a la vida subterránea a lo largo de varios linajes, en un proceso que ha tomado al menos $8 \mathrm{Ma}$ (Lessa et al. 2008). En este contexto, el estudio de adaptaciones para cavar en la anatomía del cráneo y parte del esqueleto apendicular fue ampliamente desarrollado en Ctenomyidae, pero no así en Octodontidae (Verzi 2002; Morgan y Verzi 2006; Verzi y Olivares 2006; (Lessa et al. 2008; Morgan y Verzi 2011; Morgan et al. 2017; Pérez et al. 2017).

El género Spalacopus incluye una sola especie, S. cyanus, endémica de la región central del Chile distribuida desde la costa hasta los 3,400 m en los Andes y desde la provincia de Copiapó hasta Ñuble (Pine et al. 1979; Nowak 1999; MuñozPedreros 2000). Spalacopus cyanus posee un tamaño corporal entre 80 a $120 \mathrm{~g}$ (Torres-Mura y Contreras 1998) y representa una de las especies altamente adaptada a la vida subterránea de la familia Octodontidae de la cual forma parte. A diferencia de la mayoría de los mamíferos subterráneos, $S$. cyanus es social y vive en grandes colonias con un promedio de 16 individuos hasta un máximo de 26, y comparten el uso del mismo sistema de madrigueras (Begall et al. 1999; Lacey et al. 2019).

Al igual que otros mamíferos subterráneos su cuerpo es corto y robusto, y orejas de pequeño tamaño (Torres-Mura y Contreras 1998; Verzi et al. 2015) pero a diferencia tienen especializaciones visuales diurnas resultando en una visión eficiente comparable a la de otros roedores que viven en superficie (Vega-Zuniga et al. 2017). En su morfología cráneo-dentaria posee ciertas características asociadas a la función de excavación como la intensificación de la expansión lateral del ángulo mandibular y la cresta masetérica (Vassallo y Mora 2007) y la presencia de incisivos procumbentes con los alvéolos posteriormente desplazados (Lessa et al. 2008). Por otro lado, se han realizado investigaciones sobre el tipo de suelo como un factor determinante de su morfología, en este caso se han evaluado atributos como la resistencia a la flexión y la resistencia al corte de los incisivos, pero la dureza del suelo no resultó ser un factor determinante ya que no hay diferencias significativas entre poblaciones más costeras (suelo arenoso) respecto de poblaciones de zona de montaña (suelo rocoso; Bacigalupe et al. 2002).

Si bien la morfología externa y las características cráneodentarias delaespeciese han definidoy descripto en trabajos previos (Vassallo y Verzi 2001; Bacigalupe et al. 2002; Olivares et al. 2004; Vassallo y Mora 2007; Lessa et al. 2008), la morfología postcraneal es prácticamente desconocida, sólo se han realizado estudios de la escápula, húmero y carpos (Lessa et al. 2008; Morgan 2009; Morgan y Verzi 2011). Cabe destacar que el material esquelético postcraneal de S. cyanus depositado en colecciones es escaso comparado con el material de cráneo disponible. Resulta significativo conocer en detalle la morfología del esqueleto postcraneal de esta especie poco estudiada, debido a su particular modo de vida, única dentro de los octodóntidos. El objetivo de este trabajo es describir el esqueleto postcraneal de S. cyanus e inferir aspectos funcionales de su morfología tomando otros modelos desarrollados para grupos mejores estudiados como marsupiales y algunos roedores.

\section{Materiales y métodos}

Material examinado. Para la realización de este trabajo se revisaron dos colecciones sistemáticas mastozoológicas: Museo de La Plata (MLP), La Plata, Buenos Aires, Argentina y Colección de Mamíferos del Instituto de Ciencias Ambientales y Evolutivas, Universidad Austral de Chile, Valdivia, Chile (UACH).

En total se examinaron 29 especímenes de S. cyanus con material de postcráneo completo, excepto carpos, tarsos, manos y pies. Se detallan a continuación los datos de localidad, que incluye país, provincia, departamento o comuna y localidad específica, y número de colección de los especímenes.

Chile: Choapa, Los Vilos: Los Vilos, 3 (UACH 2507, 2508, 2510). Canela: Huentelauquén, 1 (UACH 4020). Com. Quirihue, Los Remates, 2 (UACH 4017, 4018). Elqui, La Serena: Parque Nacional Fray Jorge, 1 (UACH 1844). Nuble, Con. Quirihue, Los Remates, 22 (1 MLP 10.XI. 95.5, 21 UACH 1846, $1847,2511,2512,2513,2514,2515,2516,2517,2518,4001$, 4002, 4003, 4004, 4005, 4006, 4007, 4012, 4368, 4376, 4385).

Nomenclatura y descripción del postcráneo. Para la descripción detallada de la morfología de los elementos óseos se dividió al esqueleto en las siguientes regiones: 1 ) esqueleto axial: vértebras cervicales, torácicas, lumbares, sacras y caudales, costillas y esternón. 2) Cintura escapular y miembros anteriores: clavícula, escápula, húmero, radio, ulna y carpos. 3) Cintura pélvica y miembros posteriores: 
pelvis, fémur, tibia, fíbula y tarsos. Para la descripción de cada estructura esquelética se consideró la forma, superficie, tamaño y orientación, incluyendo además la variación en número de los elementos óseos que la componen. Para las descripciones se siguió la nomenclatura propuesta por: Evans (1993), Argot (2001; 2002; 2003), Sargis (2001; 2002a, b), Horovitz y Sánchez-Villagra (2003), Bezuidenhout y Evans (2005), Morgan y Verzi (2006), Selthofer et al. (2006), Candela y Picasso (2008), Salton y Sargis $(2008 ; 2009)$, Flores (2009), Flores y Díaz (2009), Morgan (2009), Carrizo y Díaz (2011). Las estructuras del esqueleto axial fueron descriptas principalmente en sentido cráneo caudal y en los miembros en sentido próximo distal.

La observación de las estructuras se realizó con lupas estereoscópicas Leica Wild M3Z y Nikon SMZ 745T. Para una mejor visualización e ilustración de los diferentes elementos postcraneales observados se tomaron fotografías con cámara digital Nikon D3200.

\section{Resultados}

En la morfología postcraneal de S. cyanus se observan características que se consideran altamente conservadas entre los miembros de la familia Octodontidae, pero algunos caracteres son exclusivos de la especie. A continuación, se describen de manera detallada cada elemento del esqueleto postcraneal por regiones.

Esqueleto axial. Está compuesto por siete vértebras cervicales, 12 ó 13 torácicas, seis o siete lumbares, cuatro sacras, entre 16 y 18 caudales, 12 ó 13 pares de costillas y un esternón compuesto por cinco esternebras.

Atlas. Es un elemento de aspecto ovalado, su eje transversal es más largo que el dorsoventral (Figura 1a). El arco neural dorsal es corto con el margen craneal cóncavo en forma de " $U$ " y el caudal es recto o cóncavo, pero en forma de "V". El tubérculo dorsal es bajo, en cuatro especímenes apenas visible (UACH 2518, 4005, 4020, 4367). Los forámenes atlantales son redondeados y grandes, los procesos transversos son lobulados, y en vista lateral, no sobrepasan el nivel de las facetas caudales.

Las facetas craneales son más largas que anchas con forma arriñonada con los márgenes dorsales muy extendidos anteriormente. Las facetas caudales tienen forma subtriangular y se orientan caudomedialmente. Los forámenes transversos son grandes y redondeados a ovalados, pero no se observan en vista craneal debido al gran desarrollo de las facetas de articulación. El tubérculo ventral es pequeño terminando en una punta roma y orientado ventralmente.

Axis. La forma general es subtriangular, con los bordes del arco convergiendo dorsalmente en forma recta (Figura 1b). El proceso espinoso es alto y robusto, y sólo en un ejemplar (UACH 1844) se observó una extensión caudal sobrepasando la tercera vértebra cervical (C3); en vista lateral, el borde dorsal es redondeado y los bordes craneal y caudal curvos; en vista dorsal, el proceso se ensancha en dirección caudal.

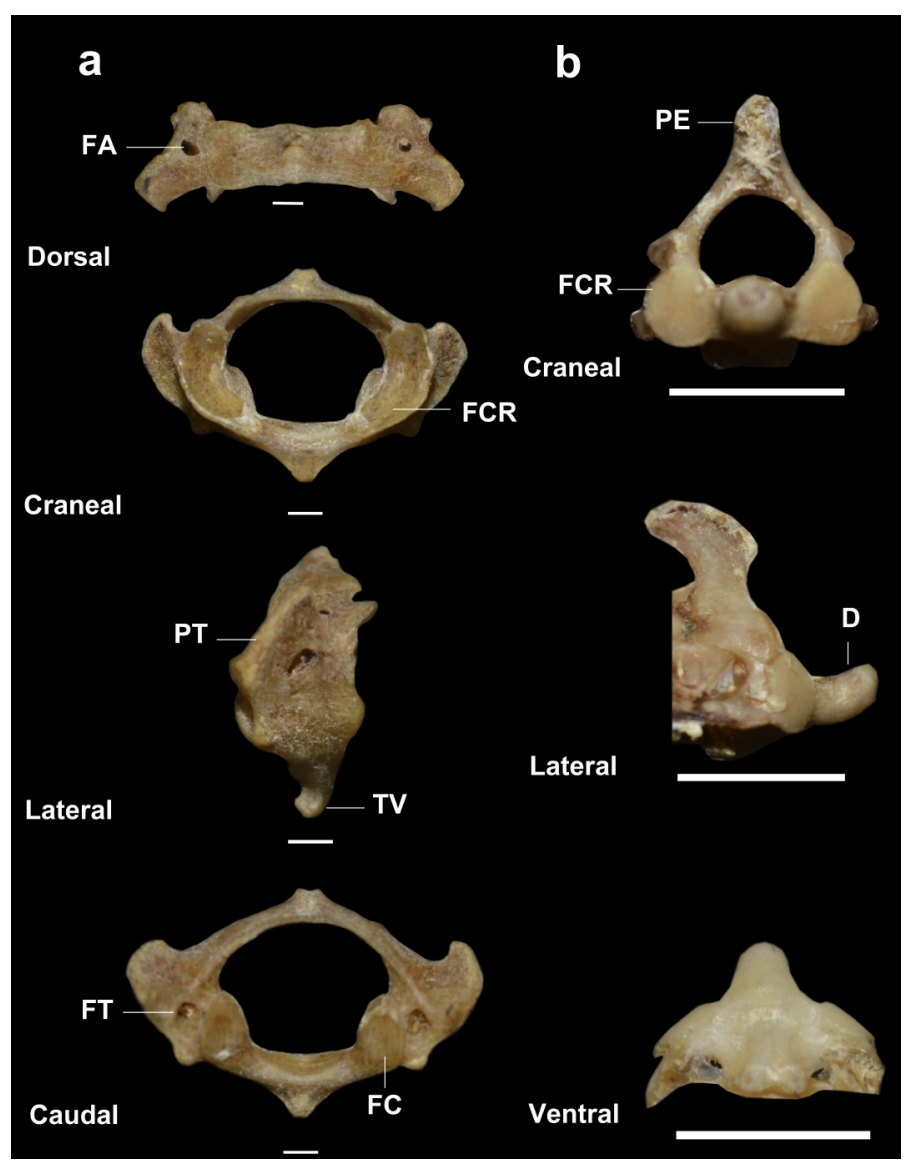

Figura 1. a) Atlas en vista dorsal, craneal, lateral y caudal. Escalas $1 \mathrm{~mm}$. b) Axis en vista craneal, lateral y ventral. Escalas $5 \mathrm{~mm}$. Abreviaturas: dens (D), forámenes atlantales $(F A)$, facetas craneales $(F C R)$, facetas caudales $(F C)$, foramen transverso $(F T)$, proceso espinoso (PE), procesos transversos (PT), tubérculo ventral (TV).

Las facetas de articulación craneal se orientan craneolateralmente y son subtriangulares. El proceso odontoides (dens) se orienta anterodorsalmente, es corto y conectado con las facetas craneales. Los procesos transversos terminan en punta y se extienden caudalmente llegando al nivel de las post-zigapófisis. Los forámenes transversos son grandes y redondeados.

Ventralmente, el arco vertebral es corto sin forámenes ventrales, el tubérculo ventral termina en forma bilobada. El borde caudal del axis articula imbricado con la C3.

Vértebras cervicales posteriores (C3-7). La forma general de las vértebras es baja y ancha. El tamaño de los arcos neurales de las $\mathrm{C} 3$ a 7 se mantienen constantes en todos los ejemplares con los bordes craneal y caudal rectos, excepto en UACH 2517 y 4002 donde el arco de la C5 es más delgado con el borde craneal cóncavo. Los espacios intervertebrales son tan anchos como el arco dorsal. Los procesos espinosos están ausentes en toda la serie cervical. Los procesos transversos son anchos y cortos desde la $\mathrm{C} 3$ a 5 y se dirigen caudalmente, en las C6 y 7 son más delgados y levemente más largos, la C6 mantiene la orientación caudal mientras que en la C7 son perpendiculares (Figura 2). Los forámenes transversos son grandes, redondeados y presentes hasta la C6. En la C6 se observa la lamela con forma de yunque invertido debido a la extensión anteroposterior de su borde más ventral, con la porción posterior más extendida en 
comparación a la extensión anterior y al arco ventral; sólo en dos ejemplares es diferente, con proyección caudal y sin extensión anterior por delante del arco ventral (UACH 2513) o con la misma extensión cráneo-caudal que el arco ventral sin proyectarse (UACH 2517).

Ventralmente, los cuerpos vertebrales muestran en las C3 y 4 un tubérculo ventral bilobado sobre el margen que contacta con la vértebra siguiente, a partir de la C5 el cuerpo es plano. No se observan forámenes en toda la serie cervical, con excepción de un ejemplar con un par pequeño en la C5 (UACH 2510). Las pre-zigapófisis se orientan dorsomedialmente con una articulación oblicua con respecto al plano sagital.

Vértebras torácicas (T1-T12 ó 13). El número de vértebras torácicas no se pudo determinar para la totalidad de los ejemplares examinados por estar la serie incompleta o por falta de limpieza de los elementos esqueléticos. En los ejemplares donde se pudo determinar el número se registraron 13 vértebras torácicas en 11 ejemplares (UACH 1846, 2514, 4002, 4003, 4005, 4006, 4012, 4017, 4018, 4376, 4385) y 12 vértebras en ocho especímenes (UACH 1844, 2510, 2513, 2518, 4001, 4020, 4367, 4368).

En vista dorsal, la forma general de las vértebras es similar a las cervicales hasta la T3, a partir de la T4 se estrechan, aumentan el largo cráneo-caudal siendo transversalmente más cortas. El proceso espinoso en la T1 es pequeño, en la T2 alcanza el máximo desarrollo, en la T3 disminuye su altura, en la T4 es más alto que la T3 aumentando gradualmente la altura hasta las T8 y 9, con algunas excepciones tales como un ejemplar (UACH 2513) donde las T3 y 4 tienen los procesos muy bajos e iguales entre sí. En relación a la orientación es vertical hasta las T3 y 4 y a partir de la T5 es caudal hasta la T9 (Figura 2). La forma, en vista lateral, tienen poca extensión cráneo-caudal hasta la T8, y en la T9 está más extendido que en las anteriores. Los procesos de las dos últimas torácicas tienen forma similar a las vértebras lumbares. Las vértebras diafragmática y anticlinal se ubican en la T10, a partir de la cual la altura del proceso espinoso se reduce notablemente e invierten su orientación en sentido craneal (Figura 2).

En la T1, los procesos transversos son latero-ventrales, desde la T2 a la T12 ó 13 los procesos transversos se van acortando y su orientación es cráneo-dorsal. Las facetas costales, donde articula el primer par de costillas, tienen forma de ventosa hasta la T9 y a partir de la T10 se reducen a una pequeña protuberancia. Dicha faceta se mantiene sobre los procesos transversos hasta la T4, a partir de la T5 se comienza a separar gradualmente hasta separarse completamente en la T8. Los procesos accesorios se observan a partir de la T9 como una proyección caudal en paralelo con el eje cráneo-caudal y en contacto con los procesos transversos; a partir de la vértebra anticlinal (T10) se separan del proceso transverso. En vista ventral, los cuerpos de las vértebras son en general planos y a partir de la T6 son levemente convexos. Uno a dos forámenes pueden estar presentes en algunas vértebras a lo largo de la serie torácica.
Vértebras lumbares (L1-6 ó 7). El número de la serie lumbar varía, al igual que con las vértebras torácicas en 11 especímenes se registran seis lumbares (UACH 1846, 2514, 4002, 4003, 4005, 4006, 4012, 4017, 4018, 4376, 4385) у en ocho (UACH 1844, 2510, 2513, 2518, 4001, 4020, 4367, 4368) se registraron siete lumbares. La primera lumbar se identifica por la ausencia de fóveas costales articulares.

Las vértebras lumbares son cuadrangulares, robustas y su tamaño aumenta hasta la L5 y luego disminuye en la L6 ó 7 (Figura 2). Los procesos son bajos y se dirigen cranealmente, pero no se extiende por delante de la articulación vertebral, sólo en la última lumbar es levemente más alto. Los procesos transversos se orientan cráneo-ventralmente con forma de aleta con la porción proximal ancha y la distal más estrecha; son cortos en las primeras lumbares y levemente más largos en las L6 y 7. Los procesos accesorios, separados de los transversos, están bien desarrollados y se observan hasta la L6. Los procesos mamilares están presentes en todas las lumbares, son muy bajos, pequeños y proyectados hasta el nivel de las facetas articulares craneales. La articulación entre la pre- y la postzigapófisis es latero-medial.

Vértebras sacras (S1-4). Todos los ejemplares examinados presentan cuatro vértebras sacras, en la mayoría de los ejemplares están fusionadas completamente entre sí desde la S1 a 3, mientras que entre las S3 y 4 la fusión es incompleta (Figura 2); sólo en un espécimen hembra la fusión no es completa entre ninguna de las vértebras sacras $(\mathrm{UACH}$ 2510). Los procesos transversos están fusionados formando una lámina. Los procesos espinosos, prácticamente en la totalidad de los ejemplares, no se fusionan entre sí, sólo en dos individuos se registra la fusión entre los procesos de la $\mathrm{S} 1$ y 2 (UACH 2513, 4020). El proceso espinoso de la S1 es el más alto y orientado cranealmente, los otros tres son perpendiculares. Las crestas sacras intermedias son evidentes.

Dorsalmente, los forámenes sacrales son pequeños y redondeados, se observan sólo dos pares debido a que la articulación entre la S3 y 4 estaba rota en todos los ejemplares examinados; el primer par es más pequeño que el segundo. Ventralmente no se observa el promontorio y se pueden observar pequeños forámenes.

Vértebras caudales (Ca1-18 ó 19). Spalacopus cyanus es una especie de octodóntido de cola corta, en la mayoría de los especímenes se registraron 19 vértebras caudales (UACH 2517, 4001, 4009, 4012, 4018, 4367), en cuatro 18 (UACH $1846,2513,2518,4006)$ y en el resto de los ejemplares la serie caudal estaba incompleta. Las dos primeras caudales son similares a la última vértebra sacra, en la Ca3 se reduce la extensión lateral de los procesos transversos y el tamaño de la vértebra (Figura 2). La articulación entre pre- y postzigapófisis se observa entre las tres primeras vértebras caudales, es reducida entre las Ca3 y 4, y a partir de la Ca4 la articulación de la porción posterior ocurre entre el cuerpo vertebral y los discos intervertebrales. Los procesos espinosos se observan bien desarrollados en las Ca1 y 2, a partir de la $\mathrm{Ca} 3$ se reducen y son visibles sólo hasta las $\mathrm{Ca} 8$ y 9 . 
Los procesos transversos están expandidos lateralmente hasta la Ca4 a partir de la cual se acortan, alcanzando la misma longitud del cuerpo vertebral en la Ca5, siendo sólo pequeñas protuberancias apenas extendidas lateralmente hasta la Ca10. En vista ventral, a partir de la Ca3 se observa el proceso hemal muy pequeño cerrado en forma de canal hasta la Ca5, a partir de la Ca6 está abierto y adquiere una forma de "H". Dichos procesos van disminuyendo en tamaño hacia las últimas vértebras y se observan hasta el final de la serie caudal conservando la forma en " $\mathrm{H}$ ".
Costillas. Once especímenes presentan 13 pares de costillas (UACH 1846, 2514, 4002, 4003, 4005, 4006, 4012, $4017,4018,4385,4376)$ y en ocho 12 (UACH 1844, 2510, $2513,2518,4001,4020,4367,4368)$. Cabe destacar que en un individuo se registró una anomalía con 13 costillas de un lado y 12 del otro (UACH 2517). El primer par de costillas es el más corto, robusto, con el borde medial cóncavo y el lateral convexo y con el extremo ventral aún más robusto y ancho. El resto de las costillas se comprimen y se vuelven progresivamente más largas, alcanzando el máximo de

a

VT

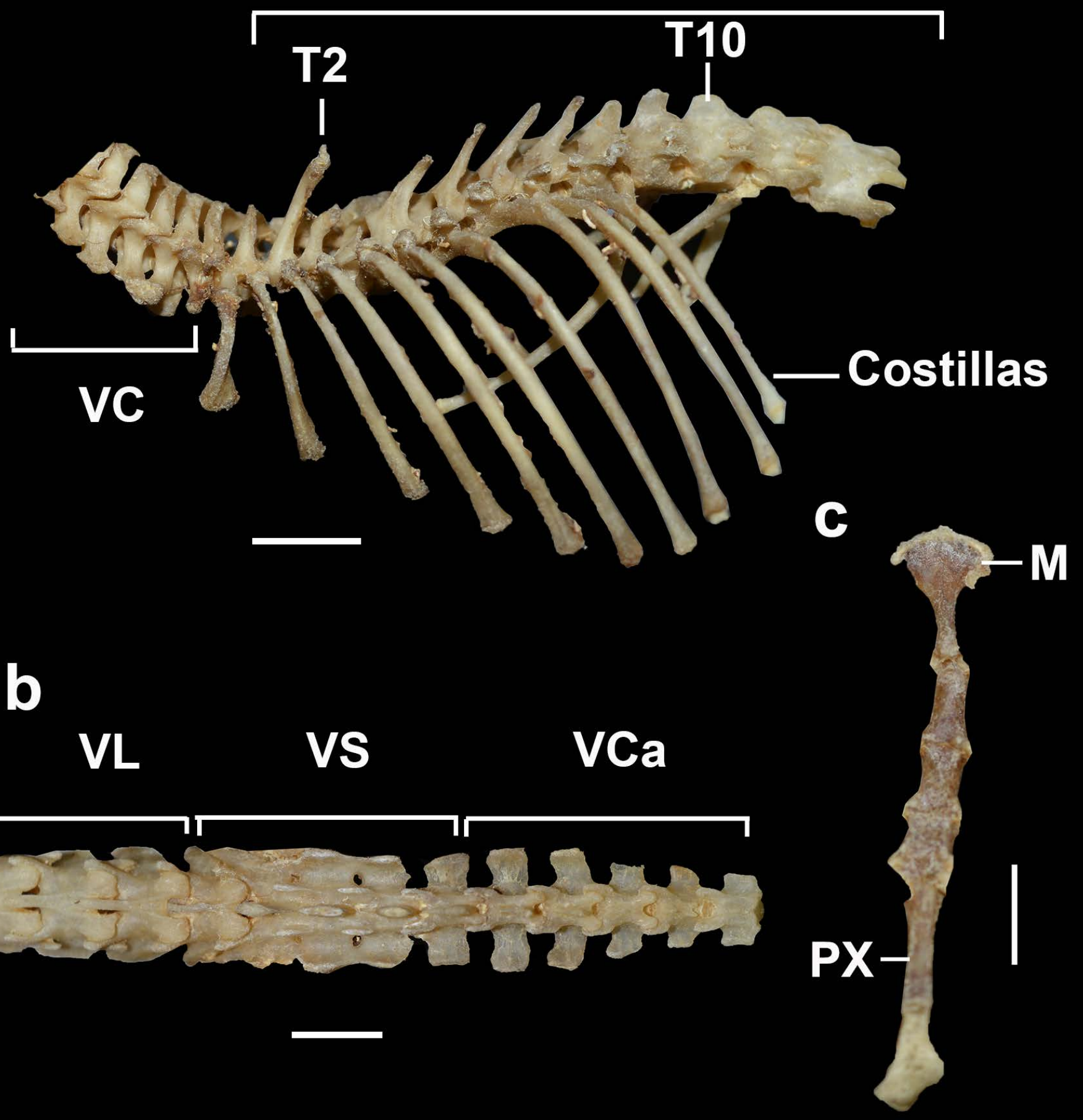

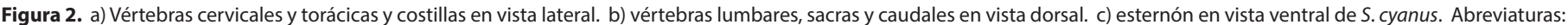

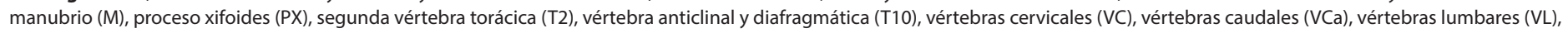
vértebras sacras (VS), vértebras torácicas (VT). Escalas $5 \mathrm{~mm}$. 
longitud en el séptimo u octavo par, y a partir de estos pares se acortan nuevamente (Figura 2). Las primeras cinco costillas presentan un cuerpo aplanado en su porción más dorsal y cilíndrico sobre su mitad ventral, hacia el final de la serie el cuerpo completo se vuelve más cilíndrico. El cuello es corto en las cuatro o cinco primeras costillas, del sexto al noveno o décimo par se alarga y en las últimas tres costillas se simplifica toda la estructura y no hay una distinción clara del cuello, entre la cabeza y el tubérculo costal. El tubérculo costal tiene su máximo desarrollo en el primer par de costillas, se mantiene bien desarrollado hasta el cuarto o quinto par y luego se reduce, pero se observa hasta el último par de costillas.

Esternón. El esternón está compuesto por cinco esternebras incluyendo manubrio y xifoides. El manubrio es la estructura de mayor tamaño y no presenta quilla, su porción anterior es aplanada en forma de abanico con el borde anterior curvo con expansiones laterales y la porción posterior es alargada y relativamente cilíndrica, lo que le da la forma de "T"; sólo en dos ejemplares las expansiones laterales de la porción anterior del manubrio son mayor y la forma se asemeja a un martillo (UACH 2510, 4002). La segunda esternebra presenta la porción anterior más angosta que la posterior, la tercera y cuarta esternebras tienen forma rectangular, estas tres son aplanadas y cortas representando aproximadamente la mitad del largo del manubrio. El xifoides es la segunda esternebra más larga, delgada y aplanada, con el cartílago xifoides en su extremo caudal, con la porción caudal extendida.

Cintura escapular y miembros anteriores; clavícula. La clavícula es delgada y sigmoidea con la curvatura más marcada hacia el extremo escapular. El extremo esternal es robusto y cilíndrico con la faceta articular esternal redondeada y el extremo escapular achatado con la faceta escapular alargada y plana.

Escápula. La escápula tiene forma triangular con los bordes craneal (o superior) y caudal (axilar o lateral) más largos que el borde vertebral (Figura 3a). La superficie lateral está dividida por la espina escapular en una fosa supraespinosa de menor tamaño que la infraespinosa. El borde craneal es sigmoideo, la curvatura es más marcada hacia el cuello de la escápula, el borde vertebral es curvo a levemente recto y el caudal es recto con un ángulo caudal más o menos evidente. La espina escapular se separa del cuerpo escapular aproximadamente a partir de la mitad posterior de la superficie lateral de la escápula, posee una leve curvatura en su extensión hacia el borde caudal y se extiende ventralmente sobrepasando la cavidad glenoidea. La espina se continúa en un ancho y romo acromion y un delgado y puntiagudo metacromion. La superficie costal presenta una leve concavidad en la mitad de su extensión sobre la línea que divide las fosas, anteroventralmente se enangosta donde se continua con el cuello que la separa de la cavidad glenoidea, esta última es ovalada. El proceso coracoides se extiende levemente en dirección antero-medial; en vista articular termina en un extremo redondeado, con una excepción observada en un ejemplar (UACH 4020) donde el proceso se encuentra más extendido y terminando en un extremo puntiagudo.

Húmero. La diáfisis es cilíndrica y recta. La cabeza humeral, en vista proximal, es redondeada terminando posteriormente en un pico caudal redondeado (Figura 3b). Los tubérculos mayor y menor no sobrepasan la altura de la cabeza humeral y están separados por un surco bicipital ancho y marcadamente cóncavo. Los tubérculos están bien desarrollados, el mayor más grande que el menor. El tubérculo mayor se ubica cráneo-lateralmente a la cabeza y es redondeado en vista lateral, sobre este tubérculo y proximalmente a la tuberosidad lateral humeral en una superficie central redonda y cóncava se inserta el $M$. infraspinatus. El tubérculo menor también es redondeado y se ubica medialmente a la cabeza. La cresta deltoidea se ubica por encima de la mitad de la diáfisis, está desplazada proximalmente y bien desarrollada, con una marcada extensión anterolateral y bordes redondeados.

En la epífisis distal, la cresta lateral epicondilar está extendida tanto lateralmente como próximo-distalmente con una curvatura evidente (Figura 3b vista distal). El foramen supratroclear está presente en la mayoría de los ejemplares y su desarrollo es variable, puede ser muy grande (UACH $1844,1846,2510,2517,2518,4001)$ o más pequeño (UACH $4005,4006,4020,4368$ ) y en tres ejemplares (UACH 2513, 4002 , 4020) está cerrado o ausente. El epicóndilo medial es mucho más grande que el lateral pero ninguno se extiende superando el borde distal de la tróclea. La tróclea presenta un mayor desarrollo próximo-distal y menor latero-medial comparado con el capítulo. La transición entre tróclea y capítulo es evidente en vista anterior, mientras que en vista posterior no se observa una división sino una única superficie en forma de polea. El capítulo tiene, en general, forma cilíndrica, aunque puede ser más redondeado; el margen lateral está engrosado, dando lugar a la cresta lateral la cual está apenas extendida por encima del borde proximal del capítulo y una cresta medial más pronunciada distalmente y orientada de manera oblicua. La fosa radial es más profunda que la del olecranon.

Radio. La diáfisis es cilíndrica, más robusta hacia el extremo distal. La articulación proximal del radio tiene forma ovalada con la superficie articular plana, el borde lateral es más redondeado que el medial con una muesca en el borde anterior. La diáfisis proximal presenta un cambio en la dirección del eje principal por debajo del nivel de la articulación proximal con la ulna; a su vez en vista anterior también se observa una marcada curvatura sobre la porción distal en dirección medial. Sobre la cara caudal medial, por debajo de la epífisis proximal, se observa la tuberosidad radial, poco desarrollada.

La epífisis distal es más robusta que la proximal, muestra una superficie articular carpal cóncava y ovalada, el eje principal está orientado craneomedialcaudolateralmente. Sobre el lado medial se observa un surco estrecho y corto próximo-distalmente para el paso del 


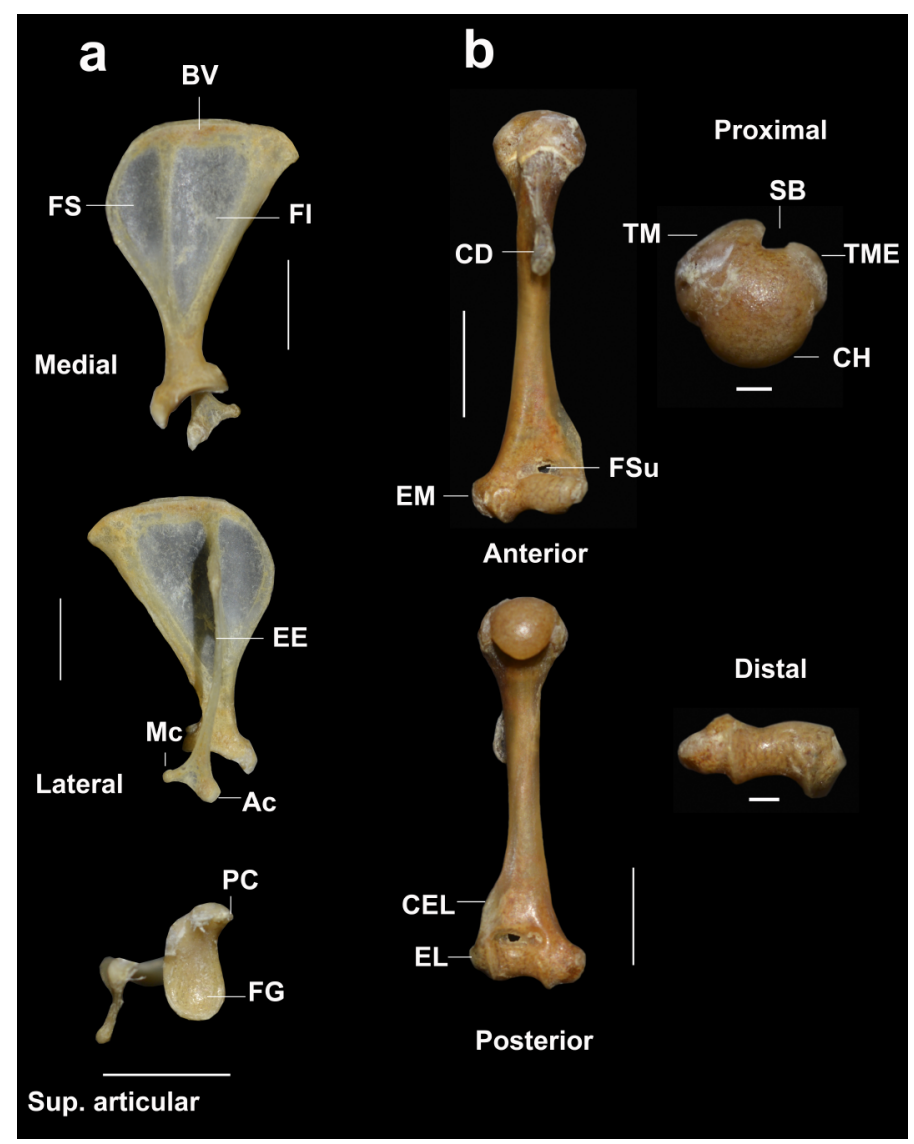

Figura 3. a) Escápula en vista medial, lateral y superficie articular. b) Húmero en vista anterior, posterior (escalas $5 \mathrm{~mm}$ ), proximal y distal (escalas $1 \mathrm{~mm}$ ). Abreviaturas: acromion $(A C)$, borde vertebral $(B V)$, cresta deltoidea $(C D)$, cresta epicondilar lateral $(\mathrm{CEL})$, cabeza humeral $(\mathrm{CH})$, espina escapular (EE), epicóndilo lateral (EL), epicóndilo medial (EM), fosa glenoidea ( $F G)$, fosa infraespinosa ( $\mathrm{FI}$ ), fosa supraespinosa ( $\mathrm{FS}$ ), foramen supratroclear (FSu), metacromion (Mc), proceso coronoides (PC), surco bicipital (SB), tubérculo mayor (TM), tubérculo menor (TME).

M. abductor digiti l longus. El proceso estiloides es pequeño, se expande latero-medialmente y apenas distalmente.

Ulna. En la epífisis proximal, el olecranon está moderadamente desarrollado cráneo-caudalmente, es relativamente largo próximo-distalmente y robusto con una zona deprimida o cóncava en la cara medial; representa, en promedio, el $17 \%$ de la longitud total de la ulna; la tuberosidad del olecranon se engrosa medialmente hasta su margen caudal con una proyección medial muy evidente. El margen craneal del olecranon es cóncavo, el proximal convexo y el caudal prácticamente recto. La muesca o incisura troclear es profunda, con forma de " $\mathrm{C}$ ". El proceso anconeal está definido por dos crestas: la cresta ulnar lateral proximal (ulptc) y la cresta ulnar medial proximal (umptc); en vista craneal, ambas crestas se observan continuas, sin distinguirse un punto de inflexión entre ambas. La umptc se orienta oblicuamente y no supera un ángulo de $30^{\circ} \mathrm{con}$ el eje próximo-distal. La ulptc tiene una gran expansión sobre la cara lateral mayor que la umptc sobre la cara medial. La muesca radial es ancha, cóncava y el eje mayor es oblicuo respecto de la diáfisis. El proceso coronoides medial está más desarrollado que el lateral, protruye anteriormente y además se proyecta medialmente en toda su extensión próximo-distal. Por debajo del proceso coronoides medial se observa una marcada fosa en vista medial proximal.
En la epífisis distal, se observan la faceta articular para el radio dispuesta medialmente y el proceso estiloides más desarrollado cráneo-caudalmente que latero-medialmente con el extremo distal redondeado y un surco sobre la cara lateral. La superficie articular se dispone disto-medialmente siendo su límite lateral el proceso estiloides propiamente dicho.

Cintura pélvica y miembros posteriores; pelvis. El ilion es más largo que el isquion, se observan alineados en vista lateral y articula con el ala de sacro (Figura 4a). El ala del ilion es larga, delgada y cóncava. El extremo anterior del ilion es recto con una leve curvatura lateral. La línea gluteal divide la superficie gluteal en una fosa gluteal (superficie gluteal dorsal) y una fosa ilíaca (superficie gluteal ventral), ambas fosas tienen un desarrollo similar, siendo la gluteal más cóncava. Cranealmente la línea gluteal se continúa lateralmente y termina en la tuberosidad coxal pobremente desarrollada dándole un aspecto redondeado al extremo craneal y caudalmente termina antes del acetábulo. Sobre el borde dorsal del ala se observa la tuberosidad sacral, levemente evidente. Caudalmente a la tuberosidad, el borde del ilion forma la incisura isquiática mayor, más larga en aquellos individuos donde se registra un ala dorsal más corta (UACH 2517, 4005, 4367, 4368).

En el cuerpo del isquion se puede observar la muesca isquiática menor, sin la presencia de una espina isquiática por delante, inclinada hacia los laterales, continuándose con los bordes caudales que se extienden marcadamente hacia los lados. La tuberosidad isquiática está bien desarrollada. La rama isquiática está curvada en forma sigmoidea.

En el cuerpo del pubis las ramas craneales se extienden dorso-lateralmente desde el cuerpo hacia la región del acetábulo y constituyen el borde craneal de la pelvis sin una eminencia iliopúbica, pero con un marcado proceso pectíneo en algunos ejemplares (UACH 2513, 2517, 4005, 4006, 4020,4367 ) y ausente en tres (UACH 4002, 4368, 4385). Las ramas caudales, derecha e izquierda, se fusionan en una línea media formando la sínfisis pélvica. La sínfisis pélvica es muy corta comparada con el foramen obturador. $Y$ en vista caudal, el arco isquiático tiene forma de " $U$ " muy abierta por la expansión lateral de los bordes caudales. El acetábulo es redondeado e interrumpido caudalmente por la incisura acetabular, la fosa acetabular es profunda y la superficie lunate está bien delimitada. La tuberosidad femoralis está ausente. El foramen obturador es ovoide, el eje cráneo-caudal tiene menor longitud comparado con el dorsoventral y el ángulo de la rama caudal es obtuso.

Se observó dimorfismo sexual en algunas estructuras de la pelvis. Las ramas caudales del pubis en las hembras son más robustas y más altas que en los machos, mientras que las craneales son más delgadas. Una particularidad registrada es la presencia de proceso pectíneo sólo en las hembras.

Fémur. El fémur es robusto con la diáfisis levemente curvada en sentido lateral en la zona media por lo que el borde medial es ligeramente cóncavo y cilíndrica en sección 
transversal (Figura 4b). En la epífisis proximal, la cabeza femoral es esférica y orientada dorso-medialmente con un cuello muy corto y un surco dorsal ancho y en " $U$ " entre el cuello y el trocánter mayor. En vista medial, la fóvea capitis se encuentra situada próxima al margen dorsal de la cabeza. Los trocánteres mayor y menor están bien desarrollados, el mayor es robusto, sobrepasa dorsalmente la altura de la cabeza y está inclinado cranealmente y en menor grado medialmente; en vista dorsal, se observa un pequeño surco en la superficie próxima al borde craneal. El trocánter menor está orientado póstero-medialmente, es de menor tamaño que el trocánter mayor y sobresale medialmente en vista caudal. En vista caudal, se observa una muy profunda fosa trocantérica que no se extiende proximalmente más allá de la altura de la cabeza y distalmente llega al nivel de la base del cuello. El tercer trocánter se localiza proximalmente sobre la diáfisis y está poco desarrollado, apenas sobresale lateralmente y en cuatro ejemplares está ausente (UACH $2517,2518,4001,4006$ ).

La epífisis distal es ancha, y la tróclea femoral se encuentra delimitada por dos crestas paralelas, medial y lateral. La superficie de la tróclea es ancha, cóncava y, en el área proximal, se extiende levemente sobre la cara craneal de la diáfisis. Los cóndilos son paralelos y separados cranealmente por la tróclea y caudalmente por una profunda y estrecha fosa intercondilar. En vista distal, el cóndilo lateral es más ancho o más extendido lateralmente que el cóndilo medial; ambos se proyectan en sentido caudal, en la mayoría de los ejemplares el medial se extiende algo más que el lateral, sólo en dos ejemplares se observan igualmente proyectados (UACH 1844, 4006). En esta vista, el desarrollo cráneo-caudal de la tróclea es mayor a la proyección caudal de los cóndilos. Las facetas para la articulación de los sesamoideos supracondiloideos están bien delimitadas.

Tibia (Figura 4c). La tibia es robusta con la porción proximal triangular en sección transversal y siendo aproximadamente un $12 \%$ más larga que el fémur. En el primer tercio proximal, la diáfisis tiene una marcada curvatura en dirección medial. En vista proximal, los cóndilos lateral y medial son piriformes y levemente cóncavos $y$, en vista anterior, están al mismo nivel. El cóndilo lateral es más ancho y cóncavo que el medial, y se continúa caudo-lateralmente con la superficie articular con la fíbula. En vista proximal, el área intercondilar craneal tiene poca extensión cráneo-caudal terminando en una tuberosidad tibial bien desarrollada. El área intercondilar caudal es estrecha y cóncava. En vista caudal, se observan sobre la diáfisis dos crestas, la lateral y una medial a esta última. La lateral se origina de la zona caudal de la diáfisis y la recorre hasta la zona de articulación con la fíbula donde se inserta el $M$. flexor digitorum lateralis. La segunda cresta es más marcada en su porción proximal y hacia la mitad de la diáfisis es más baja. La muesca poplítea es una superficie estrecha y superficial. La cresta tibial está poco desarrollada sin una evidente proyección antero-medial.
La epífisis distal presenta dos facetas astragalotibiales separadas por una cresta o elevación más evidente, son ovaladas, con la medial más ancha, corta y marcadamente más cóncava que la lateral. En vista caudal, el borde caudal es curvo con el proceso tibial distal posterior poco desarrollado, comparando con el resto de los octodóntidos es el de menor tamaño. El maléolo medial, en vista medial, es redondeado y pequeño, menos proyectado distalmente en comparación con el proceso posterior. En el material examinado no fue posible observar la presencia de surcos por el mal estado en que se encontraba del material examinado.

Fíbula (Figura 4c). La fíbula es bastante más delgada que la tibia, aplanada en su porción proximal y comprimida en toda su extensión. La cabeza articular tiene forma similar a un abanico, muy aplanada, resultando en una estructura muy simple sin surcos ni tubérculos. En la unión proximal con la tibia se observa un foramen redondeado. La fusión de la fíbula con la tibia no es ósea.

El extremo distal de la fíbula no se pudo describir en detalle por las condiciones en la que se encontraba el material examinado. El maléolo fibular lateral tiene forma bilobada, es redondeado en el punto distal de contacto con la tibia, proyectado distalmente al mismo nivel del maléolo medial.

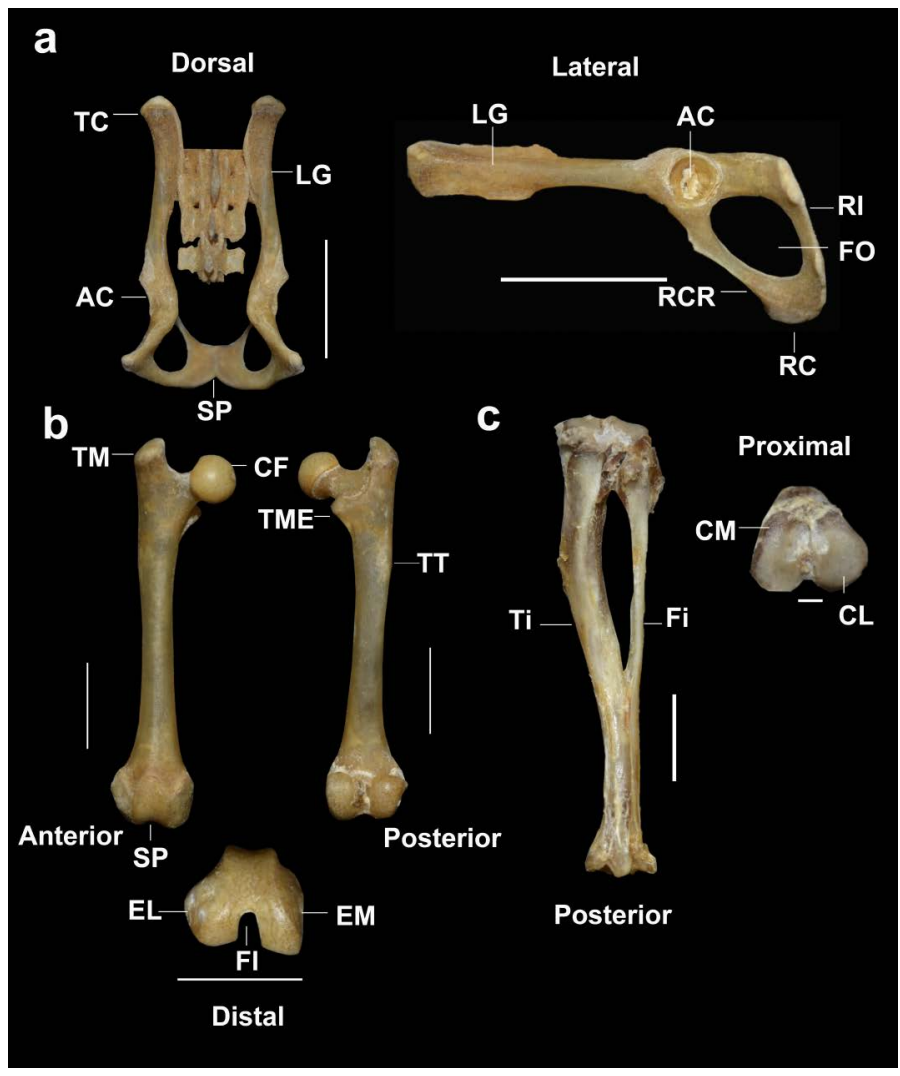

Figura 4. a) Pelvis en vistas dorsal y lateral. Escalas $10 \mathrm{~mm}$. b) Fémur en vistas anterior, posterior y distal. Escalas $5 \mathrm{~mm}$. c) Tibia y fíbula en vistas posterior y vista proximal de la tibia. Escalas $5 \mathrm{~mm}$. Abreviaturas: acetábulo (AC), cabeza femoral (CF), cóndilo medial $(\mathrm{CM})$, cóndilo lateral $(\mathrm{CL})$, epicóndilo lateral $(\mathrm{EL})$, epicóndilo medial (EM), fíbula (Fi), fosa intercondilar (FI), foramen obturador (FO), línea gluteal (LG), rama caudal $(\mathrm{RC})$, rama craneal $(\mathrm{RCR})$, rama isquiática (RI), sínfisis pélvica (SP), tuberosidad coxal (TC), tibia (Ti), trocánter mayor (TM), trocánter menor (TME), tercer trocánter (TT), surco patelar (SP). 


\section{Discusión}

En este trabajo, se describen por primera vez la mayoría de los elementos esqueléticos del postcráneo de Spalacopus cyanus, a través de dicha descripción se pudo inferir que su patrón morfológico se ajusta a un plan anatómico típico de especies terrestres. Estos resultados son consistentes con lo observado en otros mamíferos (roedores y marsupiales), como por ejemplo la ausencia de quilla en el manubrio del esternón, la extensión posterior del húmero formando un pico, los tubérculos del húmeros sin sobrepasar la cabeza humeral, la forma redondeada del acetábulo de la pelvis, la extensión del tercer trocánter por encima de la cabeza del fémur (Hatt 1932; Sargis 2002a, b; Argot 2003; Candela y Picasso 2008; Flores y Díaz 2009; Olivares 2009; Carrizo y Díaz 2011). Asimismo, en algunos elementos óseos se observan características que se corresponderían con una habilidad excavadora, principalmente en los huesos largos.

Es importante señalar que, a pesar de ser el orden más diverso de mamíferos, existen grandes faltantes de información sobre la anatomía esquelética postcraneal de los roedores. Pocos autores han realizado estudios descriptivos clásicos del postcráneo de mamíferos, pero de este tipo de investigaciones han surgido aportes muy valiosos sobre anatomía funcional, filogenia y evolución, aunque mayoritariamente en el área de paleontología (e. g., Argot 2001, 2002, 2003; Szalay y Sargis 2001; Candela y Picasso 2008; Ercoli 2015); y en menor medida sobre la mastofauna actual (e. g., Evans 1993; Bezuidenhout y Evans 2005; Flores 2009; Flores y Díaz 2009; Carrizo 2011; Carrizo y Díaz 2011; Salton y Sargis 2008, 2009; Candela et al. 2017; Gaudioso et al. 2017; Pérez et al. 2017; Pérez 2019). Muchos investigadores coinciden en afirmar que incrementar este tipo de trabajos permitirá una mejor interpretación de los atributos postcraneales funcionalmente relacionados con los diferentes estilos de vida, evaluar sus estrategias locomotoras, como así también la evolución de estos rasgos en un contexto filogenético.

A continuación, se discuten las variaciones morfológicas observadas en los elementos y en las estructuras óseas en un contexto comparativo y funcional.

Esqueleto axial. Siete vértebras cervicales y 19 toracolumbares se registran en los roedores, aunque de modo general las toracolumbares pueden presentarse como 12 y 7 ó 13 y 6 (Hatt 1932). En S. cyanus se presentan ambas combinaciones, siendo la de 12 y 7 la más frecuente.

En el atlas, el tubérculo dorsal en general está poco desarrollado e incluso ausente en algunos taxones. En esta región se insertan músculos que intervienen en movimientos rotacionales de la cabeza, tales como los Mm. obliquus capitis caudalis, splenius y rectus capitis dorsalis minor (Argot 2003; Flores y Díaz 2009); por lo tanto, en S. cyanus implicaría una capacidad limitada para los movimientos rotacionales. El tubérculo ventral poco desarrollado en esta especie limitaría la capacidad de movimiento del cuello en el plano sagital, relacionado con los músculos longus colli y longus capitis que se insertan en esta estructura (Argot 2003; Flores y Díaz 2009). Si bien la forma de sus facetas craneales con un buen desarrollo de los márgenes dorsales permitiría un rango más amplio de movimiento en la articulación atlanto-occipital, como se ha observado en algunos escandentios, marsupiales didélfidos, roedores sigmodontinos e incluso el resto de los octodóntidos (Sargis 2001; Argot 2003; Flores y Díaz 2009; Carrizo 2011; Pérez 2019).

En el axis, en vista dorsal dicho proceso tiende a ensancharse caudalmente terminando como en una " $\mathrm{V}$ " invertida o triangular, lo que proporciona una amplia superficie para la inserción del ligamento nucal, este ligamento soporta y restringe la flexión del cráneo (Sargis 2001; Argot 2003; Flores y Díaz 2009). A su vez en el proceso espinoso del axis también se insertan los músculos relacionados con los movimientos laterales y rotacionales de la cabeza (Sargis 2001; Argot 2003; Flores y Díaz 2009); y la proyección caudal de dicho proceso, si bien no es común que sobrepase la tercera cervical en esta especie, podría incrementar la ventaja mecánica de los ligamentos y músculos de esta región y actuar restringiendo los movimientos en esta región (Sargis 2001).

Los procesos espinosos en el resto de las vértebras cervicales están ausentes o levemente desarrollados, lo que restringe el área de inserción para los $\mathrm{Mm}$. spinalis cervicis y multifidus cervicis que proporcionan los movimientos en esta región y fueron particularmente relacionados con la dieta (Argot 2003; Flores y Díaz 2009), infiriendo que aquellos mamíferos con escasa movilidad del cuello (e. g., marsupiales, primates, roedores sigmodontinos) tienden a usar sus manos para llevar el alimento a la boca (Hatt 1932; Argot 2003; Carrizo 2011). Esta condición podría indicar que S. cyanus, como el resto de los octodóntidos, usan sus manos para capturar y llevar la comida a la boca, capacidad mencionada al menos para $T$. barrerae y cuyas vértebras cervicales, al igual que el resto de las especies de Tympanoctomys, carecen de procesos espinosos (Ojeda et al. 1996; Mares et al. 1997; Giannoni et al. 2000).

Los caracteres observados en esta región, hacen que esta especie tenga un movimiento del cuello restringido sumado a la incapacidad de ejercer fuerza con esta región dado a la presencia de características tales como: la orientación caudal de los procesos transversos hasta C5 y el escaso o prácticamente nulo desarrollo de la lamela inferior que se origina desde la C3 ó 4 hasta alcanzar su máximo desarrollo en C6. Las características señaladas contrastan con aquellas requeridas por animales con marcados hábitos predadores y con fuertes mordeduras (Argot 2003; Flores y Díaz 2009).

En la región torácica el proceso espinoso más alto se observa en $\mathrm{T} 2$, lugar donde se inserta el ligamento nucal y el M. splenius (Flores y Díaz 2009; Carrizo 2011), un buen desarrollo de este proceso permite el soporte y restringe los movimientos de la cabeza (ver Flores y Díaz 2009). En S. cyanus, como en los demás octodóntidos y al igual que en roedores filotinos, la vértebra anticlinal (T10-11) se observa 
en una posición anterior en la columna vertebral, lo que incrementa la fuerza de la musculatura epaxial, como consecuencia del cambio de inserción muscular sobre el ápice del proceso espinoso (Flores y Díaz 2009; Carrizo 2011; Pérez 2019); mientras que una posición anterior de la vértebra diafragmática (T10-11) indica un punto de unión anterior del M. multifidus. La posición de las vértebras anticlinal y diafragmática puede tener implicancias funcionales, ya que en estas vértebras se producen cambios relacionados con el movimiento lateral y sagital de la columna vertebral (Argot 2003; Flores y Díaz 2009). Esta ubicación en la región torácica o anterior en la columna de las vértebras anticlinal y diafragmática, puede resultar útil para los movimientos que requiere $S$. cyanus dentro del sistema de madrigueras (Reig 1989), posibilitando ciertos movimientos en el plano sagital pero con más rigidez en la flexión lateral. De igual manera no hay estudios sobre el movimiento del tronco o de la marcha de Spalacopus cyanus.

Los procesos espinosos y transversos de la región lumbar proporcionan los sitios de inserción para los músculos comprometidos con la flexión ventral de la columna y con la locomoción (Mm. quadratus lumborum y psoas mayor; Argot 2003; Flores y Díaz 2009). Cuando los procesos espinosos son bajos y largos (en referencia a la extensión dorso-ventral y cráneo-caudal de acuerdo Sargis 2001), lo que implica reducción de los espacios intervertebrales, la movilidad estaría restringida; mientras que los procesos largos y estrechos sumado a un mayor espacio intervertebral (Sargis 2001) permitirían mayor movilidad espinal. Los procesos espinosos en la región lumbar de $S$. cyanus, al igual que en el resto de los octodóntidos (Pérez 2019), muestran un patrón intermedio a lo planteado por Sargis (2001), con una extensión dorso-ventral mayor a lo establecido como procesos espinosos bajos, pero menor a lo que se considera como procesos altos, con espacios intervertebrales reducidos. Dicho patrón es similar a lo observado en otras formas cuadrúpedas con mayor flexión lateral y menor flexión y extensión sagital, típico de formas adaptadas al salto y contrario a lo que se produce en la región torácica de $S$. cyanus (ver Sargis 2001; Argot 2003). De igual manera, esta condición intermedia conferiría a los músculos extensores cierta ventaja mecánica para una extensión más poderosa durante la carrera terrestre con cierta capacidad de flexión y extensión vertebral para aumentar la longitud de zancada (Sargis 2001), sin mostrar variación desde los octodóntidos epigeos a subterráneos (Pérez 2019). A su vez, los procesos accesorios en $S$. cyanus se separan completamente del proceso transverso a partir de T10 y se mantienen hasta $L 6$, se proyectan posteriormente trabando la articulación con la vértebra siguiente, lo cual restringe la flexibilidad lateral de la columna vertebral (Argot 2003). La combinación de los caracteres expuestos, hace que $S$. cyanus tenga una columna más o menos flexible, posibilitando movimientos lateral y sagital según las diferentes etapas de la locomoción.
Spalacopus cyanus es de las pocas especies de Octodontidae con cola corta, la reducción en el número total de vértebras caudales podría relacionarse con pasar más tiempo en túneles, e incluso podría asociarse con aspectos fisiológicos para evitar la pérdida excesiva de calor (ver Ercoli 2015). La articulación entre la cuarta y quinta vértebra caudal se produce a través de los discos intervertebrales, lo que aumenta la flexibilidad de la porción proximal de la cola permitiendo movimientos laterales y según lo planteado por otros autores esta condición sugiere cierta capacidad prensil (Argot 2003; Flores y Díaz 2009; Pérez 2019), aunque esta capacidad no es propia de los octodóntidos y menos aún de un animal cavador (Pérez 2019). En cuanto a la flexibilidad de la cola para movimientos laterales, podría relacionarse con el movimiento a un lado y otro como de barrido, comportamiento observado en otros roedores que se mueven hacia atrás a través del sistema de madrigueras al igual que lo plantea Reig (1970) para $S$. cyanus y las especies de Ctenomys (ver Stein 2000). El desarrollo de los procesos transversos y espinosos en las primeras vértebras caudales indica el buen desarrollo de los $\mathrm{Mm}$. multifidus caudal, ischio-caudalis y abductor caudal dorsalis permitiendo movimiento en planos lateral y vertical, asociado con la capacidad de estabilizar al animal dentro de la madriguera (Stein 2000; Argot 2003; Flores y Díaz 2009; Carrizo 2011), y con la marcha atrás.

Costillas y esternón. La condición más común en el número de costillas es de 12 y algunos ejemplares de S. cyanus con 13 costillas; siendo estrechas en su forma no se expanden cráneo-caudalmente, morfología que se observa en formas terrestres, lo que le quitaría rigidez a la caja torácica (Sargis 2001; Argot 2003).

El esternón en Spalacopus está compuesto por cinco esternebras a diferencia del resto de los octodóntidos con seis esternebras, patrón que coincide con lo descrito para otros roedores y marsupiales (Bezuidenhout y Evans 2005; Flores y Díaz 2009; Carrizo 2011; Pérez 2019). Por otro lado, la quilla del manubrio está ausente y la presencia de esternebras comprimidas, indicaría un menor desarrollo del $M$. pectoralis y en consecuencia una potencia proporcionalmente reducida de los movimientos del tronco; patrón que coincide con lo descripto para roedores filotinos, sciúridos y marsupiales sin habilidades trepadoras (Bezuidenhout y Evans 2005; Flores y Díaz 2009; Carrizo 2011).

Cintura escapular y miembro anterior; clavícula. La clavícula juega un rol importante en la cintura escapular de los mamíferos, entre otras cosas por interconectar el esqueleto axial con el apendicular del miembro anterior (Rocha-Barbosa et al. 2002). En esta especie, tiene la forma típica de otros mamíferos (Bezuidenhout y Evans 2005; Flores y Díaz 2009) y con un desarrollo mayor comparado con roedores caviodeos donde la articulación con la escápula y el esternón se realiza por medio de ligamentos (Rocha-Barbosa et al. 2002). Su presencia mantiene una distancia fija entre el acromion y el manubrio, asegurando un movimiento muy preciso entre ambas estructuras 
(Rocha-Barbosa et al. 2002) estabilizando y dando soporte al miembro anterior.

Además, en S. cyanus, la clavícula es sólo más sigmoidea respecto de los roedores filotinos, con los extremos esternal y escapular con igual desarrollo a diferencia, por ejemplo, de los marsupiales arborícolas donde el extremo esternal está más desarrollado (Flores y Díaz 2009).

Escápula. La escápula de S. cyanus y del resto de Octodontidae tiene forma subtriangular, típica de otros roedores y algunos marsupiales (Bezuidenhout y Evans 2005; Flores y Díaz 2009; Morgan 2009; Carrizo 2011; Pérez 2019). La condición, relacionada con un patrón morfológico de formas terrestres o generalizadas $y$, de acuerdo a otros autores, a formas arborícolas (ver Salton y Sargis 2008), es un mayor desarrollo de la fosa supraespinosa (Argot 2001; Sargis 2002a; Flores y Díaz 2009) lo que proporciona mayor superficie para el $M$. supraspinatus; esto no coincide con el patrón observado en $S$. cyanus como en el resto de las especies de la familia, donde la fosa supraespinosa tiene menor desarrollo. Por otro lado, el M. infraspinatus se origina en la fosa infraespinosa y es responsable de dos funciones: abducción y rotación externa del húmero, asociado a la muesca escapular permitiendo mayor estabilidad del hombro y está menos desarrollado en las formas terrestres en comparación con arborícolas (Argot 2001); y de acuerdo a la morfología observada en S. cyanus podría ser ventajoso para excavar, si bien no es una especialización fosorial propiamente dicha (ver Morgan 2009: 503).

En todos los miembros de la familia Octodontidae se observa que el hábito locomotor no parece ser el mayor determinante de la forma escapular a nivel de especies, en coincidencia con lo planteado por Morgan 2009. Algunas diferencias o particularidades encontradas pueden asociarse con ciertas capacidades mecánicas y funcionales que se discuten a continuación. La extensión del acromion más allá del proceso coracoides, como se observa en todos octodóntidos, permite que el $M$. deltoideus envuelva el hombro por lo que el brazo de palanca es corto debido a la proximidad del centro de rotación de la articulación (Argot 2001). La longitud del acromion en un animal excavador podría indicar si el animal está generando fuerza desde la musculatura del hombro o del antebrazo (Salton y Sargis 2008), aunque mientras que en algunos cavadores el acromion está muy desarrollado, en otros el acromion está reducido (Salton y Sargis 2008). En S. cyanus, el acromion está más desarrollado que el metacromion y también más desarrollado si se compara con el acromion de otras especies de octodóntidos; pero no es tan largo como en otros cavadores más especializados (Salton y Sargis 2008), lo que indicaría que posee cierta capacidad de generar parte de la fuerza con la musculatura del hombro (Salton y Sargis 2008).

El metacromion es el sitio de inserción para los $\mathrm{Mm}$. trapezius, atlantoscapularis y omotransversarius anterior y para la extensión del M. deltoideus pars acromialis (ver Salton y Sargis 2008), los cuales son músculos involucrados con la estabilización escapular y la rotación humeral; sugiriendo que el metacromion, cuando está presente como en S. cyanus, indica el soporte de cargas pesadas en el hombro (Salton y Sargis 2008). Por lo tanto, esta especie tiene capacidad de generar fuerzas con el miembro anterior, así como también una buena estabilidad corporal.

Húmero. Algunos autores indican que la forma de la cabeza humeral no es un buen indicador de arboricoría o cursorialidad (Szalay y Sargis 2001; Candela y Picasso 2008), ya que la gran variación de este rasgo hace imposible correlacionar la forma de la cabeza con los movimientos parasagitales y rotacionales del miembro anterior con el tipo de locomoción (Candela y Picasso 2008). La cabeza humeral semiesférica de $S$. cyanus se asocia con una baja capacidad de rotación vinculado con las formas terrestres (Sargis 2002a; Argot 2003; Flores y Díaz 2009); además, se desplaza posteriormente formando un "pico" y este carácter también se encuentra asociado a formas de locomoción terrestre (Szalay y Sargis 2001). El "pico" es la estructura donde la escápula y el húmero "traban" y la articulación se vuelve más estable, a diferencia de formas arborícolas con mayor flexibilidad hacia el plano medial en esta articulación y sin un "pico" desarrollado posteriormente (Szalay y Sargis 2001).

Los tubérculos mayor y menor en $S$. cyanus no sobrepasan el nivel de la cabeza humeral, lo que permite un rango más amplio de movilidad escapular (Szalay y Sargis 2001; Candela y Picasso 2008), característica ampliamente observada en marsupiales y algunos eretizóntidos arborícolas; sin embargo, también se observa en formas terrestres de cavioideos y chinchilloideos, equímidos y octodóntidos. Debido a esta gran variabilidad, coincidimos con Candela y Picasso (2008) acerca de la imposibilidad, hasta el momento, de correlacionar la forma de la cabeza humeral con la gama de movimientos parasagitales o rotacionales de la extremidad anterior para Hystricognathi A diferencia de algunos roedores escansoriales, fosoriales y subterráneos (Stein 2000) que cuentan con una cresta deltoidea de posición más distal, S. cyanus presenta la cresta en la mitad proximal de la diáfisis lo que representaría una desventaja mecánica para los músculos deltoideo y pectoral que contribuyen a la retracción del miembro anterior (Stein 2000; Argot 2001; Morgan y Verzi 2006).

En la epífisis distal del húmero, la morfología del epicóndilo medial y de la cresta epicondilar lateral reflejan el tipo de locomoción (Argot 2001; Candela y Picasso 2008; Flores y Díaz 2009). En Spalacopus, la mayor extensión del epicóndilo medial está precisamente relacionada con la capacidad de excavar (Stein 2000; Salton y Sargis 2008), ya que la mayor extensión provee más superficie para los músculos carpales y digitales $(\mathrm{Mm}$. flexor digitorum profundus, flexor carpi radialis y flexor carpi ulnaris) que a su vez dan mayor fuerza de flexión a la muñeca y los dígitos durante la excavación (Argot 2001; Sargis 2002a). Cabe destacar que $S$. cyanus si bien usa las patas delanteras para remover el suelo mientras excava también utiliza los 
incisivos (Reig 1970). La tróclea y el capítulo marcan la superficie articular con la ulna y el radio respectivamente. La forma del capítulo se correlaciona con el movimiento del radio en el húmero, una forma esférica es indicativo de movimiento multiaxial, mientras que un capítulo trocleado se correlaciona en diversos grados con la rápida flexión o extensión de la ulna que requiere refuerzo lateral (Salton y Sargis 2008). En Spalacopus, el capítulo muestra una evidente convexidad, lo que otorgaría mayor movilidad a la unión del codo permitiendo libre rotación del radio durante la pronación y supinación del brazo, esto podría relacionarse con la capacidad de excavar (Sargis 2002a; Candela y Picasso 2008); este atributo es similar en otros dos géneros fosoriales de Octodontidae, Aconaemys y Octodon (Pérez 2019).

Radio y ulna. El radio y la ulna están relacionados con el poder de desplazamiento de la tierra y del agua ya sea al cavar o nadar (Salton y Sargis 2008). La evidente curvatura de la diáfisis del radio, que se observa en Spalacopus, como en los demás octodóntidos y algunos roedores filotinos y marsupiales, indica un mayor desarrollo de los músculos comprometidos en movimientos de pronación y supinación (Argot 2001; Carrizo 2011; Pérez 2019). Esto podría sugerir que Spalacopus, como sus congéneres, presentan buena movilidad en las manos permitiendo manipular el alimento como ya fue mencionado anteriormente y remover el sustrato.

Respecto a la forma de la cabeza del radio, existen diferentes explicaciones. En el caso de Spalacopus con una cabeza elíptica u ovalada algunos autores consideran que provee más estabilidad a la articulación húmero-radial restringiendo la rotación radial y favoreciendo el movimiento en el plano sagital, característico de mamíferos terrestres (Sargis 2002a; Candela y Picasso 2008) pero otros autores consideran que una cabeza más elíptica que redondeada caracteriza a formas excavadoras (Stein 2000).

En el olecranon se inserta el $M$. triceps brachii y se correlaciona con el comportamiento locomotor en mamíferos arborícolas, terrestres y fosoriales (Van Valkenburgh 1987; Stein 2000; Argot 2001). Cuanto más largo sea el olecranon mayor es la fuerza de palanca permitiendo una fuerte extensión del miembro anterior, característico de formas excavadoras (Salton y Sargis 2008); en Spalacopus la ulna es más robusta y el olecranon es levemente más largo que el resto de los octodóntidos (Pérez 2019).

El tamaño relativo del proceso coronoides medial y la forma y disposición de la muesca radial se correlacionan con la preferencia locomotora (Candela y Picasso 2008). Como se describe para roedores histricomorfos terrestres, como octodóntidos, el proceso coronoides medial se encuentra reducido respecto de la muesca radial (Candela y Picasso 2008). La muesca radial con una superficie ligeramente cóncava y de disposición oblicua, como se observa en Spalacopus, es característica de hábitos terrestres (Argot 2001; Candela y Picasso 2008).
Cintura pélvica y miembros posteriores; pelvis. El ilion, isquion y pubis inciden en las cambiantes cargas de los miembros posteriores durante la locomoción (Salton y Sargis 2009). La forma general y el tamaño relativo, así como las rugosidades y los tubérculos en la superficie de los tres componentes, son indicadores útiles para la interpretación funcional (Salton y Sargis 2009). En líneas generales, la pelvis de $S$. cyanus no exhibe importantes especializaciones, la morfología es bastante conservada no sólo en esta especie sino en todos los octodóntidos. La morfología del acétabulo es redondeado y abierto como en las formas terrestres, lo cual restringe la movilidad de la unión de la cadera y el movimiento en el plano parasagital, lo que incrementa la eficiencia de la locomoción terrestre (Sargis 2002b). No se observaron en los ejemplares de S. cyanus analizados, otras características asociadas a la locomoción terrestre tales como la curvatura lateral anterior del ilion, como se observa en la mayoría de los octodóntidos y que sugiere un incremento en el área de origen del músculo glutei y de la musculatura epaxial al igual que en otras formas terrestres (Argot 2002) o la presencia de la tuberosidad femoralis por delante del acetábulo que incrementaría el brazo de palanca del $M$. rectus femoris, similar a lo descrito en roedores equímidos terrestres (ver Olivares 2009).

Una característica única de Spalacopus es la forma del arco isquiático en " $U$ " con los bordes caudales muy expandidos lateralmente, a lo que hasta el momento no se le atribuye alguna condición funcional. En esta estructura se origina el complejo isquipúbico (biceps femoris, semitendinosus, semimembranosus, gracilis, adductor magnus, y quadratus femoris) desde el isquion posterior y el pubis, actuando como propulsores durante la primera etapa de carrera, cuando hay una resistencia considerable y se necesita mayor fuerza de empuje (Salton y Sargis 2009). A la vez que el complejo glúteo, que se origina en el ilion, es el principal responsable del golpe final en la propulsión de las extremidades posteriores, donde hay menos resistencia y mayor potencial de velocidad. La relación entre ambos complejos está dada por si el ilion es más largo que el isquion (como algunos mamíferos cursoriales y octodóntidos) o viceversa (algunos mamíferos acuáticos) y de acuerdo a esto, el complejo muscular que más enfatiza su acción (Salton y Sargis 2009). Otra característica descrita para mamíferos saltadores es la proyección dorsal del isquion, sin embargo, no es claro cuál sería la relación de la expansión lateral del isquion, como se observa en S. cyanus.

Fémur. Spalacopus, al igual que otros octodóntidos, muestra en la morfología del fémur atributos que se relacionan con las formas terrestres, tales como la cabeza femoral semiesférica y la extensión del trocánter mayor por encima de la cabeza femoral; lo que incrementaría el brazo de palanca y la ventaja mecánica de los Mm. glutei minimus y medius, favoreciendo así una fuerte y rápida extensión del muslo, condición descripta para otros roedores histricomorfos terrestres (Argot 2002; Candela y Picasso 2008; 
Flores y Díaz 2009, Pérez 2019). La posición del trocánter menor posteromedial, como se observó en otros roedores histricognatos terrestres, indicaría una orientación más anteroposterior de las fibras del complejo iliopsoas (Argot 2002; Candela y Picasso 2008), el cuál actuaría principalmente como un transportador del fémur, facilitando los movimientos parasagitales (Argot 2002; Candela y Picasso 2008). Además, la orientación ligeramente más caudal del trocánter menor en formas terrestres, sugiere una abducción menos poderosa y una mayor velocidad en las formas terrestres (Szalay y Sargis 2001).

Una particularidad observada en S. cyanus es la poca extensión lateral del tercer trocánter, lo que podría indicar que la extremidad posterior posee cierta habilidad de permanecer flexionada, carácter que fue mencionado para algunos escandentios arborícolas con una locomoción más lenta (Sargis 2002b). Esta característica es sólo compartida con dos especies más de su familia, $A$. fuscus y $O$. degus (Pérez 2019). Además, la posición más proximal del tercer trocánter sobre la diáfisis podría indicar una función extensora más marcada del M. gluteus superficialis similar al gluteus medius (Candela y Picasso 2008), a diferencia de lo observado en eretizóntidos y otros histricognatos terrestres donde la posición del tercer trocánter es más distal. De igual manera, la posición del tercer trocánter no está claramente relacionada con la preferencia de sustrato y la posición distal sería una condición primitiva para Hystricognathi (Candela y Picasso 2008).

En Spalacopus, la epífisis femoral distal está más comprimida anteroposteriormente comparada con los demás octodóntidos, por lo tanto, tiene una mayor extensión transversal. Esta condición indica una flexión habitual de los miembros posteriores asociado a formas arborícolas (Argot 2002; Sargis 2002b; Candela y Picasso 2008; Pérez 2019). La extensión anteroposterior de la epífisis distal determina la morfología del surco troclear (Argot 2002). Las epífisis extendidas presentan un surco troclear estrecho, largo y bien delimitado por crestas (mayoría de los octodóntidos); mientras que en las formas comprimidas el surco es ancho, poco profundo y con crestas menos marcadas (Spalacopus). Una forma similar al surco de Spalacopus se observó en géneros de tenrecoideos subterráneos y probablemente sea indicativo de abducción lateral y rotación de la tibia mientras cava y se apoya contra el sustrato (Salton y Sargis 2009). Si bien la profundidad de la rodilla brinda información sobre la ventaja mecánica de los músculos y sus comportamientos locomotores, su asociación con la preferencia de sustrato al menos para estos roedores y otros histricognatos, requiere una mayor revisión (Candela y Picasso 2008).

Otra condición que se relaciona con los hábitos locomotores es la asimetría de los cóndilos, siendo en las formas terrestres el cóndilo lateral más ancho que el medial (Argot 2002; Sargis 2002b; Candela y Picasso 2008), condición observada en Spalacopus y el resto de los octodóntidos (Pérez 2019). Además, la extensión distal de los cóndilos a la misma altura podría indicar una baja capacidad de aducción del fémur, como se observó en algunos roedores histricomorfos terrestres tales como Dolichotis, Lagostomus, Dasyprocta (Candela y Picasso 2008) y dentro de octodóntidos sólo en Spalacopus. Es importante destacar que la morfología de la epífisis distal femoral estaría más relacionada a la actividad (lenta vs. rápida) que al tipo de locomoción (terrestre vs. arborícola; ver Muizon y Argot 2003).

Tibia y fíbula. La tibia sigmoidea y levemente más larga que el fémur son condiciones descriptas como una morfología generalizada (Szalay y Sargis 2001; Argot 2002; Candela y Picasso 2008; Salton y Sargis 2009; Carrizo 2011). Este patrón se describe para S. cyanus y demás octodóntidos, aunque sólo en esta especie el espacio entre la tibia y la fíbula es mayor debido a una curvatura más evidente de la tibia, esto proporciona mayor superficie para el músculo flexor digitorum fibularis (ver Flores y Díaz 2009). Esto último fue descrito para marsupiales arborícolas, y en el caso de estos roedores no está muy clara su función; de acuerdo a Szalay y Sargis (2001) no es probable que estas características sean indicativo confiable de los atributos funcionales que se pueden vincular a las condiciones ecológicas, particularmente la preferencia de sustratos o modos locomotores. Existen diversas teorías acerca de la curvatura de los huesos largos como la tibia, pero todas difíciles de entender como resultados de un equilibrio entre fuerzas femorocrurales, ya que tanto la presencia como ausencia de curvatura en la tibia se observa en mamíferos terrestres, escansoriales y arborícolas (Szalay y Sargis 2001).

Los cóndilos tibiales son asimétricos en Splacopus, el lateral más grande y más cóncavo que el medial, dicha asimetría les confiere mayor estabilidad en la articulación; este patrón morfológico fue observado en marsupiales terrestres y arborícolas, como en otros roedores histricognatos (Candela y Picasso 2008; Flores y Díaz 2009; Pérez 2019).

El M. quadriceps femoris se inserta directamente en la rótula, que a su vez se une a la tuberosidad tibial por el ligamento patelar $y$, por lo tanto, la tuberosidad tibial que se proyecta anteriormente aumenta la ventaja mecánica de este grupo muscular y permite una rápida extensión de la rodilla (Candela y Picasso 2008; Salton y Sargis 2009). Este desarrollo de la tuberosidad tibial se observa en formas terrestres de marsupiales y roedores (Szalay y Sargis 2001; Argot 2002; Candela y Picasso 2008; Flores y Díaz 2009; Carrizo 2011). En Spalacopus, al igual que los restantes octodóntidos, se observa una proyección anterior de la tuberosidad tibial. El conocimiento respecto a la variación de la forma de la tuberosidad tibial dentro del grupo de roedores histricognatos es escaso, algunos autores sostienen que es necesario estudiar en detalle la posible asociación con las habilidades cursoriales, cavadoras y saltadoras (Candela y Picasso 2008).

En la región distal de la tibia de Spalacopus y el resto de los octodóntidos (Pérez 2019), se observa que la superficie articular de la tibia para la tróclea astragalar está más extendida medio-lateralmente que antero-posteriormente 
lo que le otorga cierta movilidad a la articulación (Candela y Picasso 2008). Aunque, en coincidencia con marsupiales y otros histricognatos terrestres, los movimientos laterales estarían restringidos debido a que la superficie articular de la tibia presenta una faceta lateral cóncava separada de la faceta medial por una cresta más o menos evidente (Argot 2002; Candela y Picasso 2008).

La fíbula, es una estructura muy reducida no sólo en Spalacopus sino que en todos los miembros de Octodontidae, siendo una estructura muy sencilla con el extremo proximal en forma de abanico, características asociadas a una locomoción terrestre (Szalay y Sargis 2001; Argot 2002; Pérez 2019). Debido a que su función se limita a la transmisión de cargas compresivas longitudinales, su estructura delgada no es un inconveniente (Argot 2002). Además, la diáfisis de la fíbula no se curva ni es robusta por lo tanto los músculos que allí se originan, como el flexor digitorum fibularis, están reducidos y en consecuencia su función prensil, extensión y flexión del pie (Argot 2002; Flores y Díaz 2009; Carrizo 2011).

La mayoría de los roedores subterráneos usan el miembro posterior para sostener su cuerpo dentro de las madrigueras, mover el cuerpo hacia adelante, arrojar los desechos hacia atrás, retroceder en las madrigueras, entre otras actividades (Stein 2000). La unión de la tibia y fíbula genera una estructura más reforzada para desarrollar las actividades antes mencionadas, pero es importante destacar que este carácter por sí mismo no es un indicativo de un estilo de vida subterráneo (Stein 2000).

Para concluir, la morfología del postcráneo de Spalacopus cyanus exhibe características consistentes con algunas habilidades para excavar en determinados elementos, y otras con habilidades claramente coherentes con el hábito epigeo. Si bien la morfología funcional del postcráneo se está analizando en otros órdenes de mamíferos, incluso otros grupos de roedores, las descripciones detalladas y sus interpretaciones funcionales permanecen inconclusas para gran parte del orden Rodentia. Esta información permitiría mejorar las interpretaciones de adaptación morfológica para determinados movimientos en relación a la diversidad de hábitos locomotores como también cambios en la postura. Por otro lado, también se podrían ampliar las explicaciones a un contexto filogenético en busca de convergencias o apomorfías que permitan enriquecer los estudios de historia evolutiva tanto de representantes actuales como fósiles. En el caso particular de Spalacopus resulta interesante dilucidar el camino que esta especie tuvo hacia la vida subterránea, ampliando la información disponible de estos animales poco estudiados y enriqueciendo comparaciones con otros roedores tanto emparentados como de linajes más distantes. En referencia a lo anteriormente mencionado, la comparación con Ctenomys, roedor subterráneo y emparentado con octodóntidos, podría aportar resultados interesantes, más aún cuando la descripción de la morfología de sus elementos postcraneales es muy escasa y hasta nula si se considera el esqueleto axial.

\section{Agradecimientos}

Agradecemos a D. H. Verzi, G. D'Elia y F. Mondaca por permitirnos el acceso al material de la colección y por su asistencia y colaboración (MLP, La Plata, Buenos Aires y UACH, Valdivia, Chile). Este trabajo fue realizado gracias al aporte de una beca doctoral otorgada por CONICET (Consejo Nacional de Investigaciones Científicas y Técnicas).

\section{Literatura citada}

ARgot, C. 2001. Functional-Adaptive anatomy of the forelimb in the Didelphidae, and the paleobiology of the Paleocene Marsupials Mayulestes ferox and Pucadelphys andinus. Journal of Morphology 247:51-79.

ARgot, C. 2002. Functional-Adaptive analysis of the hindlimb anatomy of extant Marsupials and Paleobiology of the Paleocene Marsupials Mayulestes ferox and Pucadelphys andinus. Journal of Morphology 253:76-108.

ARGot, C. 2003. Functional-adaptative anatomy of the axial skeleton of some extant marsupials and the paleobiology of the Paleocene marsupials Mayulestes ferox and Pucadelphys andinus. Journal of Morphology 255:279-300.

Bacigalupe, L. D., J. IriaRTe- Díaz, Y F. Bozinovic. 2002. Functional morphology and geographic variation in the digging apparatus of cururos (Octodontidae: Spalacopus cyanus). Journal of Mammalogy 83:145-52.

Begall, S., Burda, H., Y M. H. Gallardo. 1999. Reproduction, postnatal development and growth of social coruros, Spalacopus cyanus (Octodontidae, Rodentia) from Chile. Journal of Mammalogy 80:210-217.

Bezuidenhout, A. J., y H. E. Evans. 2005. Anatomy of woodchuck (Marmota monax). Special publicación №13. American Society of Mammalogist. Kansas, EE.UU.

Candela, A. M., N. A. Muñoz, y C. M. García-Esponda. 2017. The tarsal-metatarsal complex of caviomorph rodents: Anatomy and functional-adaptive analysis. Journal of Morphology 278:828-847.

Candela, A., Y M. B. J. Picasso. 2008. Functional Anatomy of the limbs of Erethizontidae (Rodentia, Caviomorpha): indicators of locomotor behavior in Miocene Porcupines. Journal of Morphology 269:552-593.

Carrizo, L. V. 2011. Revisión sistemática y filogenética de la tribu Phyllotini (Rodentia: Sigmodontinae). Tesis de doctorado no publicada. Facultad de Ciencias Naturales e Instituto Miguel Lillo, Universidad Nacional de Tucumán.

Carrizo, L. V., y M. M. Díaz. 2011. Descripción del postcráneo de Rhipidomys austrinus y Graomys griseoflavus (Rodentia, Cricetidae, Sigmodontidae). Iheringia 101:207-219.

Ercol, M. D. 2015. Morfología del aparato músculoesqueletario del postcráneo de los mustélidos (Carnivora, Mammalia) fósiles y vivientes de América del Sur: implicancias funcionales en un contexto filogenético. Tesis de doctorado no publicada. Facultad de Ciencias Naturales y Museo, Universidad Nacional de La Plata.

Evans, H. E. 1993. Miller's Anatomy of the dog, $3^{\text {rd }}$ edition. Saunders Company. Philadelphia, EE.UU.

Fernandes, F. A., R. Fornel, P. Cordeiro-Estrela, y T. R. O. Freitas. 2009. Intra- and interspecific skull variation in two sister species of the subterranean rodent genus Ctenomys (Rodentia, 
Ctenomyidae): coupling geometric morphometrics and chromosomal polymorphism. Zoological Journal of the Linnean Society 155:220-237.

Flores, D. A. 2009. Phylogenetic Analyses of postcranial skeletal morphology in Didelphid marsupials. Bulletin of the American Museum of Natural History 320:1-81.

Flores, D. A., y M. M. Díaz. 2009. Postcranial skeleton of Glironia venusta (Didelphimorpha, Didelphidae, Caluromyinae): Descripcion and functional morphology. Zoosystematic and Evolution 85:311-339.

GallaRDo, M. H. 1992. Karyotypic evolution in octodontid rodents based on C-band analysis. Journal of Mammalogy 73:89-98.

Gallardo, M. H. 1997. A saltational model of karyotypic evolution in the Octodontoidea (Mammalia, Rodentia). Pp 347-365 en: Chromosom (Henriques-Gil, T.N., J.S. Parker, y M.J. Puertas, eds). Chapman and Hall, London, Reino Unido.

Gallardo, M.H., y F. Mondaca. 2002. The systematics of Aconaemys (Rodentia, Ocotodontidae) and the distribution of A. sagei in Chile. Mammalian Biology 67:105-112.

Gallardo, M. H., Y J. W. A. KIRSCH. 2001. Molecular relationships among Octodontidae Mammalia: Rodentia: Caviomorpha). Journal of Mammalian Evolution 8:73-89.

Gallardo, M. H., G. Kausel, A. Jiménez, C. Bacquet, C. González, J. Figueroa, N. Köhler, y R. A. OJeda. 2004. Wholegenome duplications in South American desert rodents (Octodontidae). Biological Journal of the Linnean Society 82:443-451.

Gallardo, M. H., C. A. González, y I. Cebrian. 2006. Molecular cytogenetics and allotetraploidy in the red vizcacha rat, Tympanoctomys barrerae (Rodentia, Octodontidae). Genomics 88:214-221.

Gallardo, M. H., R. A. Ojeda, C. A. González y C. A. Ríos. 2007. The Octodontidae revisited. Pp. 695-720 in The quintessential naturalist. Honoring the life and legacy of Oliver P. Pearson (Kelt, D. A., E. Lessa, J. Salazar-Bravo y J. L. Patton, eds.). Press Publications in Zoology 134. CaliforniaBerkeley, EE.UU.

Gaudioso, P. J., M. M Díaz, y R. M. Barquez. 2017. Morphology of the axial skeleton of seven bat genera (Chiroptera: Phyllostomidae). Anais da Academia Brasileira de Ciências 89: 2341-2358

Giannoni, S. M., C. E. Borghi, y R. A. OJeda. 2000. Feeding behaviour of Tympanoctomys barrerae, a rodent specialized in consuming Atriplex leaves. Journal of Arid Environments 46:117-121.

HatT, T. R. 1932. The vertebral columns of Ricochetal Rodents. Bulletin of The American Museum of Natural History 63:599-738.

Honeycutt, R. L., D. L. Rowe, y M. H. Gallardo. 2003. Molecular systematics of the South American caviomorph rodents: relationships among species and genera in the family Octodontidae. Molecular Phylogentics and Evolution 26:476-489.

Horovitz, I. y M. R. SÁnchez-Villagra. 2003. A morphological analysis of marsupial mammal higher-lever phylogenetic relationships. Cladistics 19:181-212.

Köhler, N., M. H. Gallardo, L. C. Contreras, y J. C. Torres-Mura. 2000. Allozymic variation and systematic relationships of the Octodontidae and allied taxa (Mammalia, Rodentia). Journal of Zoology 252:243-250.
Lacey, E. A., S. L. O'Brien, R. Sobrero, y L. A. Ebensperger. 2019. Spatial relationships among free-living cururos (Spalacopus cyanus) demonstrate burrow sharing and communal nesting. Journal of Mammalogy 100:1918-1927.

Lessa, E. P., A. I. Vassallo, D. H. Verzi, y M. S. Mora. 2008. Evolution of morphological adaptations for digging in living and extinct ctenomyid and octodontid rodents. The Linnean Society of London, Biological Journal of the Linnean Society 95:267-283.

Mares, M. A., J. K. Braun, R. M. Barquez, y M. M. Díaz. 2000. Two new genera and species of halophytic desert mammals from isolated Salt Flats in Argentina. Occasional Papers, Museum of Texas Tech University 203:1-27.

Mares, M. A., R. A. Ojeda, C. E. Borghi, S. M. Giannoni, G. B. Diaz, y J. K. Braun. 1997. How desert rodents overcome halophytic plant defenses. BioScience 47: 699-704.

Morgan, C. C. 2009. Geometric morphometrics of the scapula of South American caviomorph rodents (Rodentia: Hystricognathi): Form, function and phylogeny. Mammalian Biology 74:497-506.

Morgan, C. C., y D. H. Verzi. 2006. Morphological diversity of the humerus of the South American subterranean rodent Ctenomys (Rodentia, Ctenomyidae). Journal of Mammalogy 87:1252-1260.

Morgan, C. C., Y D. H. Verzi. 2011. Carpal-metacarpal specializations for burrowing in South American octodontoid rodents. Journal of Anatomy 219:167-175.

Morgan, C. C., D. H. Verzi, A. I. Olivares, y E. C. Vieytes. 2017. Craniodental and forelimb specializations for digging in the South American subterranean rodent Ctenomys (Hystricomorpha, Ctenomyidae). Mammalian Biology 87:118-124.

Muizon, C., y C. ARgot. 2003. Comparative anatomy of the Tiupampa didelphimorphs; an approach to locomotory habits of early marsupials. Pp. 43-62 in Predators with pouches: the biology of carnivorous marsupials (Jones, M. E., C. R. Dickman, y M. Archer, eds.). Csiro publishing. Acton, Australia.

Muñoz- Pedreros, A. 2000. Orden Rodentia. Pp. 73-106 en Mamíferos de Chile (Muñoz- Pedreros, A., y J. YáñezValenzuela, eds.). Valdivia: CEA Ediciones.

Nowak, R. M. 1999. Walker's mammals of the World. $6^{\text {th }}$ ed. Johns Hopkins University Press. Baltimore, EE.UU.

OJeda, A. A., A. Novillo, R. A. OJeda y S. Roig-Juñent. 2013. Geographical distribution and ecological diversification of South American octodontid rodents. Journal of Zoology 289: 285-293.

OJeda R. A., J. M. Gonnet, C. E. Borghi, S. M. Giannoni, C. M. CAmpos, Y G. B. DiAz. 1996. Ecological observations of the red vizcacha rat, Tympanoctomys barrerae in desert habitats of Argentina. Mastozoología Neotropical 3:183-191.

Olivares, A. I. 2009. Anatomía, Sistemática y Evolución de los roedores caviomorfos sudamericanos del género Eumysops ameghino (Rodentia, Echimyidae). Tesis doctoral no publicada, Facultad de Ciencias Naturales y Museo, Universidad Nacional de la Plata.

Olivares, A. I., D. H. Verzi, y A. I. Vassallo. 2004. Masticatory morphological diversity and chewing modes in South American caviomorph rodents (family Octodontidae). Journal of Zoology 263:167-177. 
Opazo, J. C., M. P. Bugueño, M. J. Carter, R. E. Palma, y F. Bozinovic. 2008. Phylogeography of the subterranean rodent Spalacopus cyanus (Caviomorpha, Octodontidae). Journal of Mammalogy 89:837-844.

Pérez, M. J. 2019. Estudio de la forma, función y tamaño de los elementos esqueletales de la familia Octodontidae (Mammalia: Rodentia) en un contexto filogenético. Tesis doctoral no publicada, Facultad de Ciencias Naturales e Instituto Miguel Lillo, Universidad Nacional de Tucumán.

Pérez, M. J., R. M. Barquez, y M. M. Díaz. 2017. Morphology of the limbs in the semi-fossorial desert rodent species of Tympanoctomys (Octodontidae, Rodentia). ZooKeys 710:77-96.

Pine, R. H., S. D. Miller, y M. L. Schamberger. 1979. Contributions to the mammalogy of Chile. Mammalia 43:339-76.

Reig, O. A. 1970. Ecological Notes on the fossorial octodont rodent Spalacopus cyanus (Molina). Journal of Mammalogy 51:592-601.

ReIG, O. A. 1989. Karyotypic repatterning as one triggering factor in cases of explosive speciation. Pp. 246-289 in Evolutionary Biology of Transient Unstable Populations. (Fontdevila, A., ed.). Springer-Verlag. Berlin, Alemania.

Rocha-Barbosa, O., D. Youlatos, J.-P. Gasc, y S. Renous. 2002. The clavicular region of some cursorial Cavioidea (Rodentia: Mammalia). Mammalia 66:413-421.

Salton, J. A., y E. J. SARGis. 2008. Evolutionary Morphology of the Tenrecoidea (Mammalia) Forelimb Skeleton. Pp. 51-71 in Mammalian Evolutionary Morphology: A Tribute to Frederick S. Szalay (Sargis, E.J., y M. Dagosto, eds.). Vertebrate Paleobiology and Paleoanthropology Series. Springer. Cham, Suiza.

Salton, J. A., y E. J. SaRGis. 2009. Evolutionary Morphology of the Tenrecoidea (Mammalia) Hindlimb Skeleton. Journal of Morphology 270:367-387.

SARGIS, E. J. 2001. The grasping behaviour, locomotion and substrate use of the tree shrews Tupaia minor and T. tana (Mammalia, Scandentia). Journal of Zoology 253:485-490.

SARGIS, E. J. 2002a. Functional morphology of the forelimb of tupaiids (Mammalia, Scandentia) and its phylogenetic implications. Journal of Morphology 253:10-42.

SarGIS, E. J. 2002b. Functional morphology of the hindlimb of tupaiids (Mammalia, Scandentia) and its phylogenetic implications. Journal of Morphology 254:149-185.

Selthofer, R. V. Nikolic, T. Mrcela, R. Radic, I. Leksan, I. Rudez, y K. Selthofer. 2006. Morphometric analysis of the sternum. Collegium Antropologicum 30:43-47.

Stein, B. R. 2000. Morphology of subterranean rodents. Pp 19-61 in Life Underground: The Biology of Subterranean Rodents (Lacey, E. A., J. L. Patton, y G. N. Cameron, eds.). Chicago, IL: University of Chicago Press. Chicago, EE.UU.

Suarez-Villota, E. Y., C. A. González-Wevar, M. H. Gallardo, R. A. VÁsquez, y E. Poulin. 2016. Filling phylogenetic gaps and the biogeographic relationships of the Octodontidae (Mammalia: Hystricognathi). Molecular Phylogenetics and Evolution 105:96-101.

Szalay, F. S., y E. Sargis. 2001. Model-based analysis of postcranial osteology of marsupials from the Paleocene of Itaboraí. Brazil, and the phylogenetic and biogeography of Metatheria. Geodiversitas 23:139-302.

Torres-Mura, J. C., y L. C. Contreras. 1998. Spalacopus cyanus. Mammalian Species 594:1-5.
Upham, N. S., y B. D Patterson. 2012. Diversification and biogeography of the Neotropical caviomorph lineage Octodontoidea (Rodentia: Hystricognathi). Molecular Phylogenetics and Evolution 63:417-429.

Upham, N. S., y B. D. Patterson. 2015. Evolution of caviomorph rodents: a complete phylogeny and timetree for living genera. Pp. 63-120 in Biology of caviomorph rodents: diversity and evolution (Vassallo, A.I., y D. Antenucci, eds.). SAREM Series A-Mammalogical Research. Buenos Aires, Argentina.

Van Valkenburgh, B. 1987. Skeletal indicators of locomotor behavior in living and extinct carnivores. Journal of Vertebrate Paleontology 7:162-182.

Vassallo, A. I., y D.H. Verzı. 2001. Patrones morfológicos craneanos y modalidades de masticación en roedores caviomorfos. Boletín de la Sociedad de Biología de Concepción 72:139-145.

Vassallo, A. I., y M. S. Mora. 2007. Interspecific scaling and Ontogenetic growth patterns of the skull in living and fossil ctenomyid and Octodontid rodents (Caviomorpha: Octodontoidea). Pp. 945-968 in The quintessential naturalist: honoring the life and legacy of Oliver P. Pearson (Kelt, D. A., E. P. Lessa, J. Salazar-Bravo, y J. L Patton, eds.) Publisher: Special Publications of The University of California Press. California, EE.UU.

Vega-Zuniga, T., F. Medina, y G. Marín, J. C. Letelier, A.G. Palacios, P. Němec, C. E. Schleich, y J. Mpodozis. 2017. Selective binocular vision loss in two subterranean caviomorph rodents: Spalacopus cyanus and Ctenomys talarum. Science Report 7:1-12.

Verzl, D. H. 1994. Origen y Evolución de los Ctenomyinae (Rodentia, Octodontidae): un análisis de anatomía cráneodentaria. Tesis Doctoral no publicada, Biblioteca Florentino Ameghino. Facultad de Ciencias Naturales y Museo, Universidad Nacional de La Plata, La Plata, Buenos Aires.

Verzl, D. H. 2002. Patrones de evolución morfológica en Ctenomyinae (Rodentia, Octodontidae). Mastozoología Neotropical 9:309-328.

Verzi, D. H. y A.I. Olivares. 2006. Craniomandibularjoint in South American burrowing rodents (Ctenomyidae): adaptations and constraints related to a specialized mandibular position in digging. Journal of Zoology 270:488-501.

Verzi, D. H., A. I. Olivares, y C. C. Morgan. 2014. Phylogeny and evolutionary patterns of South American octodontoid rodents. Acta Palaeontológica Polonica 59:757-769.

Verzi, D. H., M. M. Díaz, y R. M. Barquez. 2015. Family Octodontidae. Pp. 1023-1024 in Mammals of South America. Volume 2, Rodents: (Patton, J. L., U. F. J. Pardiñas, y G. D’Elía, eds.) University of Chicago Press, Chicago, EE.UU.

Associated editor: Jorge Salazar Bravo

Submitted: April 14, 2020; Reviewed: May 3, 2020;

Accepted: August 29, 2020; Published on line: September 11, 2020. 